\title{
Diffusion models for Knudsen compressors
}

$\operatorname{AUTHOR}(\mathrm{S})$ :

Aoki, Kazuo; Degond, Pierre; Takata, Shigeru; Yoshida, Hiroaki

\section{CITATION:}

Aoki, Kazuo ...[et al]. Diffusion models for Knudsen compressors. PHYSICS OF FLUIDS 2007, 19(11): 117103.

ISSUE DATE:

2007-11

URL:

http://hdl.handle.net/2433/84589

\section{RIGHT:}

Copyright 2007 American Institute of Physics. This article may be downloaded for personal use only. Any other use requires prior permission of the author and the American Institute of Physics. 


\title{
Diffusion models for Knudsen compressors
}

\author{
Kazuo Aoki \\ Department of Mechanical Engineering and Science and Advanced Research Institute of Fluid Science \\ and Engineering, Graduate School of Engineering, Kyoto University, Kyoto 606-8501, Japan
}

Pierre Degond

Mathématiques pour l'Industrie et la Physique, Université Paul Sabatier, 118, Route de Narbonne, 31062 Toulouse cedex, France

\section{Shigeru Takata}

Department of Mechanical Engineering and Science and Advanced Research Institute of Fluid Science and Engineering, Graduate School of Engineering, Kyoto University, Kyoto 606-8501, Japan

\author{
Hiroaki Yoshida \\ Department of Aeronautics and Astronautics, Graduate School of Engineering, Kyoto University, \\ Kyoto 606-8501, Japan
}

(Received 8 April 2007; accepted 11 July 2007; published online 8 November 2007)

\begin{abstract}
A rarefied gas in a long straight pipe with a periodic structure consisting of alternately arranged narrow and wide pipes and with periodic temperature distribution, which is known as the Knudsen compressor (or pump), is considered. Under the assumption that the pipe is much thinner than the period, a diffusion model that describes the pressure distribution and mass flux of the gas in each pipe element is derived, together with the connection conditions at the junctions of the narrow and wide pipes, from the kinetic system composed of the Boltzmann equation and its boundary condition on the pipe wall. Then, on the basis of the diffusion model and the connection conditions, a global diffusion model for the entire pipe is constructed by means of homogenization. The present work is a formal extension to the case of the Boltzmann equation of the previous work [K. Aoki and P. Degond, Multiscale Model. Simul. 1, 304 (2003)], where a simplified version of the BhatnagarGross-Krook (BGK) collision model is used. Some numerical results based on the original BGK model are also presented both for the global diffusion model and for the local diffusion model with the connection conditions at the junctions. (C) 2007 American Institute of Physics.
\end{abstract}

[DOI: $10.1063 / 1.2798748]$

\section{INTRODUCTION}

Kinetic theory of gases (or molecular gas dynamics) $)^{1-5}$ plays increasingly important roles in microfluidics. ${ }^{6-9}$ Thermally driven flows, which are peculiar to rarefied gases (or gases in the kinetic regime), have potential applicability as nonmechanical flow controllers. As the thermally driven flows, mention should be made of the thermal creep flow (thermal transpiration), ${ }^{10-14}$ thermal stress slip flow, ${ }^{15,16}$ nonlinear thermal stress flow, ${ }^{16,17}$ and thermal edge flow. ${ }^{18,19}$ The last flow, which is induced around a sharp edge when it is heated (or cooled) compared with the circumstances, was discovered rather recently by a numerical simulation ${ }^{18,19}$ and was observed experimentally. ${ }^{19}$ A thermal pump using this flow (thermal edge pump) was proposed and fabricated recently. ${ }^{20}$ On the other hand, the thermal creep flow or thermal transpiration, which is a flow along the nonuniformly heated wall in the direction of the gradient of the temperature, is a classical phenomenon that has been investigated by many scientists. ${ }^{10-14}$ A notable application of this flow is the Knudsen pump (or compressor) and its variants. ${ }^{21-32}$

The typical Knudsen pump is a long pipe with a periodic structure consisting of alternately arranged narrow and wide pipes. The temperature of the pipe is also periodic with the same period as the structure, such as a sawtooth distribution increasing in the narrow segments and decreasing in the wide segments. Such a pipe causes a unidirectional gas flow with a pumping effect. The flow and its pumping effect have been studied numerically ${ }^{24,28}$ by the direct simulation Monte Carlo (DSMC) method $^{33,34}$ as well as experimentally. ${ }^{27,29,30} \mathrm{In}$ practical applications, however, a large number of segments should be used, so that the estimate of the properties and performance of the pump in various steady and unsteady situations by the DSMC computation or by experiment is not an easy task. Therefore, if we have a simple macroscopic model of the pump, it would be very useful.

For this reason, considering the case where the linear dimension (e.g., the diameter) of the cross section of the pipe is much smaller than the length of the segments, Aoki and Degond $^{35}$ derived a diffusion model for each segment with a connection condition at the junctions of the segments with different cross sections. Further, on the basis of this diffusion model, they derived, by homogenization, a diffusion model that describes the overall behavior of a gas in a long pipe composed of a large number of the segments. As the basic kinetic equation, a Bhatnagar-Gross-Krook (BGK) type equation is employed for the purpose of obtaining the diffusion coefficients in the model equation in an analytical form. However, the BGK-type equation is not a standard one for rarefied gas flows but a special one for gas flows through a background with a given temperature distribution. 
Therefore, in the present study, we try to derive corresponding diffusion models on the basis of the true BGK model in the kinetic theory of gases. ${ }^{36,37}$ But, except for the concrete numerical examples, the analysis is carried out for the general Boltzmann equation. Because of this generalization, we need to assume some properties that could be proven in Ref. 35. In addition, the diffusion coefficients in the diffusion model are not obtained analytically. These coefficients are essentially given by the mass-flow rate of the Poiseuille flow and that of the thermal transpiration flow in an infinitely long pipe for the whole range of the Knudsen number. Fortunately, a database, based on the BGK model, for these quantities constructed by Sone and Itakura ${ }^{38}$ is available for a circular pipe and a channel between two infinite plates, so that we can exploit it for the actual numerical computations.

In Sec. II, we consider a rarefied gas in an infinitely long straight pipe with constant cross section. The temperature of the pipe, which is uniform in a cross section, is distributed arbitrarily in the axial direction of the pipe. Assuming that the dimension of the cross section is much smaller than the length scale of variation of the axial distribution of the wall temperature, we derive, from the Boltzmann equation, a diffusion-type equation that describes the global mass flow rate as well as the pressure and temperature (or density) distributions along the pipe. The result is an extension of that in Ref. 35 to the Boltzmann equation. In Sec. III, we consider the case where two semi-infinite straight pipes with different uniform cross sections are connected. We derive the connection condition at the junction of the two pipes for the diffusion model derived in Sec. II. Again, the procedure is essentially the same as that of Ref. 35. In Sec. IV, we consider a long pipe with periodic structure, repetition of a narrow and a wide segment, with a periodic temperature distribution. This is a basic structure of the Knudsen pump. As in Ref. 35, we apply the diffusion model and the connection condition derived in Secs. II and III to this Knudsen pump. Here, the homogenization technique is employed to construct an overall diffusion model for the entire Knudsen pump. In Sec. V, some numerical examples based on the diffusion model and the connection condition derived in Secs. II and III are shown. Numerical results based on the diffusion model derived by homogenization in Sec. IV are also presented and compared with the former results. The last Sec. VI is a short concluding remark.

We remark here that diffusion models corresponding to those in Secs. II and III, based on Ref. 35 and along the same line as the present paper, have been derived for a gas mixture (in the case of a two-dimensional channel) in the study of gas separation by means of the Knudsen pump. ${ }^{39}$

\section{FLOW INDUCED IN A LONG PIPE WITH A CONSTANT CROSS SECTION}

\section{A. Problem and basic equation}

Let us consider a rarefied gas in a long straight pipe with a constant cross section. We take the $X_{3}$ axis along the pipe and let the cross section be denoted by the domain $\mathrm{S}$ in the $\left(X_{1}, X_{2}\right)$ plane with area $S$. The temperature of the pipe wall, which is constant in time and uniform in the plane $X_{3}$ = const, has a distribution described by $T_{w}\left(X_{3}\right)$. The characteristic length of the cross section $\mathrm{S}$ is denoted by $D$, and the scale of variation of $T_{w}\left(X_{3}\right)$ by $L$. We investigate the flow of the gas induced in the pipe under the following assumptions:

(i) The behavior of the gas is described by the Boltzmann equation.

(ii) The characteristic length of the cross section is much smaller than the scale of variation of the temperature of the pipe wall, i.e., $D \ll L$.

The Boltzmann equation reads

$$
\partial_{t} f+\boldsymbol{\xi} \cdot \nabla_{X} f=J(f, f)
$$

where $t$ is the time variable, $\mathbf{X}$ (or $X_{i}$ ) is the position vector in the physical space, $\boldsymbol{\xi}$ (or $\xi_{i}$ ) is the molecular velocity, $f(t, \mathbf{X}, \boldsymbol{\xi})$ is the velocity distribution function of the gas molecules, $\nabla_{X}$ is the gradient operator with respect to $\mathbf{X}$, and $J(f, f)$ is the collision integral defined through the following bilinear operator (see Refs. 2 and 4):

$$
\begin{aligned}
& J(g, h)= \frac{1}{2 m} \int\left[g\left(\boldsymbol{\xi}_{*}^{\prime}\right) h\left(\boldsymbol{\xi}^{\prime}\right)+g\left(\boldsymbol{\xi}^{\prime}\right) h\left(\boldsymbol{\xi}_{*}^{\prime}\right)-g\left(\boldsymbol{\xi}_{*}\right) h(\boldsymbol{\xi})\right. \\
&\left.-g(\boldsymbol{\xi}) h\left(\boldsymbol{\xi}_{*}\right)\right] B(|\mathbf{e} \cdot \mathbf{V}| / V, V) d \Omega(\mathbf{e}) d \boldsymbol{\xi}_{*}, \\
& \boldsymbol{\xi}^{\prime}=\boldsymbol{\xi}+(\mathbf{e} \cdot \mathbf{V}) \mathbf{e}, \quad \boldsymbol{\xi}_{*}^{\prime}=\boldsymbol{\xi}_{*}-(\mathbf{e} \cdot \mathbf{V}) \mathbf{e}, \\
& \mathbf{V}=\boldsymbol{\xi}_{*}-\boldsymbol{\xi}, \quad V=|\mathbf{V}|,
\end{aligned}
$$

where $g$ and $h$ are functions of the molecular velocity $\boldsymbol{\xi}, m$ is the mass of a molecule, $B$ is a nonnegative function whose functional form depends on molecular models (e.g., for hardsphere molecules, $B=d_{m}^{2}|\mathbf{e} \cdot \mathbf{V}| / 2$ with $d_{m}$ being the diameter of a molecule), e is a unit vector, $\boldsymbol{\xi}_{*}$ is the integration variable for $\boldsymbol{\xi}, d \Omega$ is the solid-angle element around $\mathbf{e}, d \boldsymbol{\xi}_{*}$ $=d \xi_{*_{1}} d \xi_{* 2} d \xi_{* 3}$, and the domain of integration is all the directions of $\mathbf{e}$ and the whole space of $\boldsymbol{\xi}_{*}$.

The boundary condition for the Boltzmann equation on the pipe wall is written as

$$
f(t, \mathbf{X}, \boldsymbol{\xi})=\int_{\boldsymbol{\xi}_{*} \cdot \mathbf{n}<0} K_{B}\left(t, \mathbf{X} ; \boldsymbol{\xi}, \boldsymbol{\xi}_{*}\right) f\left(t, \mathbf{X}, \boldsymbol{\xi}_{*}\right) d \boldsymbol{\xi}_{*},
$$$$
\text { for } \boldsymbol{\xi} \cdot \mathbf{n}>0 \text {, }
$$

where $\mathbf{n}$ is the unit normal vector to the pipe wall, pointing to the gas, and $K_{B}$ is a scattering kernel satisfying the following conditions (see Refs. 2 and 4; the arguments $t$ and $\mathbf{X}$ in $K_{B}$ are omitted here):

(a) Positivity,

$$
K_{B}\left(\boldsymbol{\xi}, \boldsymbol{\xi}_{*}\right) \geq 0, \quad \text { for } \boldsymbol{\xi} \cdot \mathbf{n}>0 \quad \text { and } \boldsymbol{\xi}_{*} \cdot \mathbf{n}<0 .
$$

(b) Normalization,

$$
\int_{\xi \cdot \mathbf{n}>0} \frac{\boldsymbol{\xi} \cdot \mathbf{n}}{\left|\boldsymbol{\xi}_{*} \cdot \mathbf{n}\right|} K_{B}\left(\boldsymbol{\xi}, \boldsymbol{\xi}_{*}\right) d \boldsymbol{\xi}=1, \quad \text { for } \boldsymbol{\xi}_{*} \cdot \mathbf{n}<0
$$


(c) Reciprocity,
$\begin{aligned} \boldsymbol{\xi} \cdot \mathbf{n} K_{B}\left(\boldsymbol{\xi}, \boldsymbol{\xi}_{*}\right) M_{w}\left(\boldsymbol{\xi}_{*}\right)= & \left|\boldsymbol{\xi}_{*} \cdot \mathbf{n}\right| K_{B}\left(-\boldsymbol{\xi}_{*},-\boldsymbol{\xi}\right) M_{w}(\boldsymbol{\xi}), \\ & \text { for } \boldsymbol{\xi} \cdot \mathbf{n}>0 \text { and } \boldsymbol{\xi}_{*} \cdot \mathbf{n}<0 .\end{aligned}$

Here, $M_{w}(\xi)$ is a Maxwellian distribution defined in terms of the temperature $T_{w}\left(X_{3}\right)$ of the pipe wall as

$M_{w}(\boldsymbol{\xi})=\frac{\sigma}{\left(2 \pi R T_{w}\right)^{3 / 2}} \exp \left(-\frac{|\boldsymbol{\xi}|^{2}}{2 R T_{w}}\right)$,

where $\sigma$ is arbitrary and $R$ is the gas constant per unit mass $(R=k / m$ with $k$ the Boltzmann constant).

The condition (b) leads to the impermeability condition on the boundary,

$$
\int \boldsymbol{\xi} \cdot \mathbf{n} f d \boldsymbol{\xi}=0
$$

Here and in what follows, the domain of integration with respect to $\boldsymbol{\xi}$ is the whole space unless the contrary is stated. From conditions (b) and (c), it follows that $M_{w}$ satisfies Eq. (3). We assume that other Maxwellians do not satisfy Eq. (3). This assumption excludes specular reflection. Hereafter, we assume that $K_{B}$ is independent of $t, X_{1}$, and $X_{2}$ and depends on $X_{3}$ only through $T_{w}$, i.e., $K_{B}=K_{B}\left(T_{w}\left(X_{3}\right) ; \boldsymbol{\xi}, \boldsymbol{\xi}_{*}\right)$. We also assume that the kernel $K_{B}$ is locally isotropic, ${ }^{4}$ i.e., it is invariant for a rotation around $\mathbf{n}$ and for the reflection with respect to a plane containing $\mathbf{n}$. In the case of complete accommodation (or diffuse reflection), $K_{B}$ is given as

$$
K_{B}\left(T_{w} ; \boldsymbol{\xi}, \boldsymbol{\xi}_{*}\right)=-\frac{1}{2 \pi\left(R T_{w}\right)^{2}} \boldsymbol{\xi}_{*} \cdot \mathbf{n} \exp \left(-\frac{|\boldsymbol{\xi}|^{2}}{2 R T_{w}}\right) .
$$

In addition to the boundary condition, an initial condition should be prescribed,

$$
f(0, \mathbf{X}, \boldsymbol{\xi})=f^{0}(\mathbf{X}, \boldsymbol{\xi}) .
$$

The macroscopic quantities, such as the density $\rho$, flow velocity $\mathbf{v}$ (or $v_{i}$ ), pressure $p$, and temperature $T$ of the gas, are given by the following moments of $f$ :

$$
\begin{aligned}
& \rho=\int f d \boldsymbol{\xi}, \\
& \mathbf{v}=\frac{1}{\rho} \int \boldsymbol{\xi} f d \boldsymbol{\xi}, \\
& p=R \rho T=\frac{1}{3} \int|\boldsymbol{\xi}-\mathbf{v}|^{2} f d \boldsymbol{\xi} .
\end{aligned}
$$

\section{B. Scaling}

Let us seek the solution whose length scale of variation is $L$ in the direction along the pipe $\left(X_{3}\right.$ direction) and $D$ in the cross section of the pipe. For this purpose, we introduce the following dimensionless variables:

$$
\begin{aligned}
& \hat{t}=t / t_{*}, \quad\left(x_{1}, x_{2}\right)=\left(X_{1}, X_{2}\right) / D, \quad x_{3}=X_{3} / L, \quad \zeta=\xi /\left(2 R T_{*}\right)^{1 / 2}, \\
& \hat{f}=f /\left[\rho_{*}\left(2 R T_{*}\right)^{-3 / 2}\right], \quad \hat{\rho}=\rho / \rho_{*},
\end{aligned}
$$

$\hat{\mathbf{v}}=\mathbf{v} /\left(2 R T_{*}\right)^{1 / 2}, \quad \hat{T}=T / T_{*}$,

$\hat{p}=p / p_{*}, \quad \hat{T}_{w}=T_{w} / T_{*}$,

with

$$
p_{*}=R \rho_{*} T_{*} .
$$

Here, $t_{*}, \rho_{*}, T_{*}$, and $p_{*}$ are the reference time, the reference density, the reference temperature, and the reference pressure, respectively; $\rho_{*}$ and $T_{*}$ are arbitrary, whereas $t_{*}$ will be specified below. In what follows, the position vector $\left(x_{1}, x_{2}, x_{3}\right)$ is denoted by $\mathbf{x}$, a two-dimensional vector is denoted by an underscore, i.e., $\underline{\mathbf{x}}=\left(x_{1}, x_{2}\right)$ and $\underline{\boldsymbol{\zeta}}=\left(\zeta_{1}, \zeta_{2}\right)$, and $\nabla_{\underline{x}}$ indicates the gradient operator with respect to $\underline{\mathbf{x}}$.

Then, the Boltzmann equation is recast as

$$
S_{*} \partial_{\hat{f}} \hat{f}+\underline{\zeta} \cdot \nabla_{\underline{x}} \hat{f}+\delta \zeta_{3} \partial_{x_{3}} \hat{f}=\frac{2}{\sqrt{\pi}} \frac{1}{K_{*}} \hat{J}(\hat{f}, \hat{f}),
$$

where

$$
\begin{aligned}
& \hat{J}(g, h)=\frac{1}{2} \int\left[g\left(\boldsymbol{\zeta}_{*}^{\prime}\right) h\left(\boldsymbol{\zeta}^{\prime}\right)+g\left(\boldsymbol{\zeta}^{\prime}\right) h\left(\boldsymbol{\zeta}_{*}^{\prime}\right)-g\left(\boldsymbol{\zeta}_{*}\right) h(\boldsymbol{\zeta})\right. \\
& \left.\quad-g(\boldsymbol{\zeta}) h\left(\boldsymbol{\zeta}_{*}\right)\right] \hat{B}(|\mathbf{e} \cdot \hat{\mathbf{V}}| \hat{V}, \hat{V}) d \Omega(\mathbf{e}) d \boldsymbol{\zeta}_{*}, \\
& \boldsymbol{\zeta}^{\prime}=\boldsymbol{\zeta}+(\mathbf{e} \cdot \hat{\mathbf{V}}) \mathbf{e}, \quad \boldsymbol{\zeta}_{*}^{\prime}=\boldsymbol{\zeta}_{*}-(\mathbf{e} \cdot \hat{\mathbf{V}}) \mathbf{e}, \\
& \hat{\mathbf{V}}=\boldsymbol{\zeta}_{*}-\boldsymbol{\zeta}, \quad \hat{V}=|\hat{\mathbf{V}}|, \\
& d \boldsymbol{\zeta}_{*}=d \zeta_{* 1} d \zeta_{\zeta_{2}} d \zeta_{* 3}, \\
& \hat{B}=B / B_{*}, \\
& B *=\int M\left(\boldsymbol{\xi}_{*}\right) M(\boldsymbol{\xi}) B(|\mathbf{e} \cdot \mathbf{V}| / V, V) d \Omega(\mathbf{e}) d \boldsymbol{\xi}_{*} d \boldsymbol{\xi} \\
& M(\boldsymbol{\xi})=\frac{1}{\left(2 \pi R T_{*}\right)^{3 / 2}} \exp \left(-\frac{|\boldsymbol{\xi}|^{2}}{2 R T_{*}}\right) .
\end{aligned}
$$

The parameters $S_{*}, K_{*}$, and $\delta$ in Eq. (14) are defined by

$$
S_{*}=\frac{D}{t_{*}\left(2 R T_{*}\right)^{1 / 2}}, \quad K_{*}=\frac{l_{*}}{D}, \quad \delta=\frac{D}{L},
$$

where $l_{*}$ is the mean free path of the gas molecules in the equilibrium state at rest with density $\rho_{*}$ and temperature $T_{*}$, i.e.,

$$
l_{*}=\frac{2}{\sqrt{\pi}} \frac{\left(2 R T_{*}\right)^{1 / 2}}{\left(\rho_{*} / m\right) B_{*}} .
$$

It should be noted that $B_{*}$ diverges in general, unless an appropriate cutoff, such as the angular cutoff, of the intermolecular potential is introduced (see Ref. 39 for a modification of $B_{*}$ in the noncutoff case). For hard-sphere molecules, $B_{*}$ is 
given by $B_{*}=4 \sqrt{\pi} d_{m}^{2}\left(R T_{*}\right)^{1 / 2}$, so that $\hat{B}=|\mathbf{e} \cdot \hat{\mathbf{V}}| / 4 \sqrt{2 \pi}$ and $l_{*}=\left[\sqrt{2} \pi d_{m}^{2}\left(\rho_{*} / m\right)\right]^{-1}$. The $S_{*}$ and $K_{*}$ are, respectively, the Strouhal number and the Knudsen number for our reference equilibrium state. It should be noted that $\hat{B}$ in general depends on the reference temperature $T_{*}$ (it is independent of $T_{*}$ for hard-sphere molecules). ${ }^{4}$

The dimensionless version of the boundary condition is written as

$$
\begin{aligned}
\hat{f}(\hat{t}, \mathbf{x}, \zeta)= & \int_{\boldsymbol{\zeta}_{*} \cdot \mathbf{n}<0} \hat{K}_{B}\left(\hat{T}_{w}\left(x_{3}\right) ; \zeta, \zeta_{*}\right) \hat{f}\left(\hat{t}, \mathbf{x}, \zeta_{*}\right) d \boldsymbol{\zeta}_{*}, \\
& \text { for } \boldsymbol{\zeta} \cdot \mathbf{n}>0, \quad \underline{\mathbf{x}} \in \partial \mathrm{S},
\end{aligned}
$$

where $\partial \mathrm{S}$ is the location of the pipe wall in the $\left(x_{1}, x_{2}\right)$ plane and

$$
\hat{K}_{B}\left(\hat{T}_{w}\left(x_{3}\right) ; \boldsymbol{\zeta}, \boldsymbol{\zeta}_{*}\right)=\left(2 R T_{*}\right)^{3 / 2} K_{B}\left(T_{w}\left(X_{3}\right) ; \boldsymbol{\xi}, \boldsymbol{\xi}_{*}\right),
$$

and that of the initial condition is

$$
\hat{f}(0, \mathbf{x}, \zeta)=\hat{f}^{0}(\mathbf{x}, \zeta)
$$

where $\hat{f}^{0}(\mathbf{x}, \zeta)=\left(2 R T_{*}\right)^{3 / 2} \rho_{*}^{-1} f^{0}(\mathbf{X}, \boldsymbol{\xi})$.

In general, $\hat{K}_{B}$ depends on the reference temperature $T_{*}$. In the case of the diffuse reflection, it is independent of $T_{*}$ and is given as

$$
\hat{K}_{B}=-\frac{2}{\pi} \frac{1}{\hat{T}_{w}^{2}} \zeta_{*} \cdot \mathbf{n} \exp \left(-\frac{|\zeta|^{2}}{\hat{T}_{w}}\right) .
$$

The definition of the macroscopic quantities (11) becomes

$$
\begin{aligned}
& \hat{\rho}=\int \hat{f} d \zeta, \\
& \hat{\mathbf{v}}=\frac{1}{\hat{\rho}} \int \zeta \hat{f} d \zeta, \\
& \hat{p}=\hat{\rho} \hat{T}=\frac{2}{3} \int|\boldsymbol{\zeta}-\hat{\mathbf{v}}|^{2} \hat{f} d \zeta .
\end{aligned}
$$

Because of the assumption of a thin pipe [assumption (ii) in Sec. II A], $\delta$ is a small parameter. In this situation, the flow induced in the channel by the temperature field is expected to be small. If we assume that the flow speed is of the order of $\left(2 R T_{*}\right)^{1 / 2} \delta$ (this assumption is consistent with our analysis below), the appropriate reference time may be $L /\left(2 R T_{*}\right)^{1 / 2} \delta$, and thus the resulting Strouhal number is $\delta^{2}$, i.e.,

$$
\begin{aligned}
t_{*} & =\frac{L}{\left(2 R T_{*}\right)^{1 / 2} \delta}=\frac{L^{2}}{\left(2 R T_{*}\right)^{1 / 2} D}, \\
S_{*} & =\delta^{2} .
\end{aligned}
$$

On the other hand, we assume that $K_{*}$ is of the order of unity. In summary, the equation to be solved is

$$
\delta^{2} \partial_{\hat{f}} \hat{f}+\underline{\zeta} \cdot \nabla_{\underline{x}} \hat{f}+\delta \zeta_{3} \partial_{x_{3}} \hat{f}=\frac{2}{\sqrt{\pi}} \frac{1}{K_{*}} \hat{J}(\hat{f}, \hat{f}) .
$$

It should be noted that, for a given shape of the cross section of the pipe, the solution to Eqs. (24) and (18) generally depends on the reference temperature $T_{*}$ in addition to the dimensionless parameters $\delta$ and $K_{*}$. It is independent of $T_{*}$ if hard-sphere molecules (or the BGK model) are employed and the diffuse-reflection boundary condition is assumed.

\section{Asymptotic analysis and diffusion model}

In this subsection, we try to obtain the solution to Eq. (24) subject to the boundary condition (18) in the form of expansion in the small parameter $\delta$,

$$
\hat{f}=\hat{f}_{(0)}+\hat{f}_{(1)} \delta+\hat{f}_{(2)} \delta^{2}+\cdots .
$$

Correspondingly, the macroscopic quantities are expanded as

$$
\hat{h}=\hat{h}_{(0)}+\hat{h}_{(1)} \delta+\hat{h}_{(2)} \delta^{2}+\cdots,
$$

where $\hat{h}$ represents $\hat{\rho}, \hat{\mathbf{v}}, \hat{p}$, and $\hat{T}$. The relation between $\hat{f}_{(m)}$ and $\hat{h}_{(m)}$ is obtained by inserting the expansions (25) and (26) in Eq. (22) and equating the coefficients of the same power of $\delta$. Substitution of Eq. (25) into Eqs. (24) and (18) leads to a sequence of boundary-value problems, which can be solved successively from the lowest order.

\section{Zeroth order in $\delta$}

The equation and the boundary condition for the zeroth order in $\delta$ are as follows:

$$
\begin{aligned}
\underline{\boldsymbol{\zeta}} \cdot \nabla_{\underline{x}} \hat{f}_{(0)}= & \frac{2}{\sqrt{\pi}} \frac{1}{K_{*}} \hat{J}\left(\hat{f}_{(0)}, \hat{f}_{(0)}\right), \\
\hat{f}_{(0)}(\hat{t}, \mathbf{x}, \boldsymbol{\zeta})= & \int_{\boldsymbol{\zeta}_{*} \cdot \mathbf{n}<0} \hat{K}_{B}\left(\hat{T}_{w} ; \boldsymbol{\zeta}, \boldsymbol{\zeta}_{*}\right) \hat{f}_{(0)}\left(\hat{t}, \mathbf{x}, \boldsymbol{\zeta}_{*}\right) d \boldsymbol{\zeta}_{*}, \\
& \text { for } \boldsymbol{\zeta} \cdot \mathbf{n}>0, \underline{\mathbf{x}} \in \partial \mathrm{S} .
\end{aligned}
$$

The variables $\hat{t}$ and $x_{3}$ enter the above system only as parameters. The corresponding macroscopic quantities $\hat{\rho}_{(0)}, \hat{\mathbf{v}}_{(0)}$, $\hat{T}_{(0)}$, and $\hat{p}_{(0)}$ are expressed as

$$
\begin{aligned}
& \hat{\rho}_{(0)}=\int \hat{f}_{(0)} d \zeta, \\
& \hat{\mathbf{v}}_{(0)}=\frac{1}{\hat{\rho}_{(0)}} \int \zeta \hat{f}_{(0)} d \zeta, \\
& \hat{p}_{(0)}=\hat{\rho}_{(0)} \hat{T}_{(0)}=\frac{2}{3} \int\left|\zeta-\hat{\mathbf{v}}_{(0)}\right|^{2} \hat{f}_{(0)} d \zeta .
\end{aligned}
$$

It is easily seen that a solution to Eqs. (27) and (28) is given by the following Maxwellian independent of $\underline{\mathbf{x}}$, which is the dimensionless counterpart of $M_{w}$ of Eq. (7):

$$
\hat{f}_{(0)}=\frac{\sigma_{(0)}\left(\hat{t}, x_{3}\right)}{\left[\pi \hat{T}_{w}\left(x_{3}\right)\right]^{3 / 2}} \exp \left(-\frac{|\zeta|^{2}}{\hat{T}_{w}\left(x_{3}\right)}\right),
$$

where $\sigma_{(0)}$ is an arbitrary (dimensionless) function of $\hat{t}$ and $x_{3}$. It can also be shown that this is the unique solution within 
the arbitrariness of $\sigma_{(0)}$ (Appendix A). Substitution of Eq. (30) into Eqs. (29a)-(29c) yields

$$
\begin{aligned}
& \hat{\rho}_{(0)}=\sigma_{(0)}, \quad \hat{\mathbf{v}}_{(0)}=0, \\
& \hat{T}_{(0)}=\hat{p}_{(0)} / \hat{\rho}_{(0)}=\hat{T}_{w}\left(x_{3}\right) .
\end{aligned}
$$

Thus, the zeroth-order solution is written as

$$
\hat{f}_{(0)}=\frac{\hat{\rho}_{(0)}\left(\hat{t}, x_{3}\right)}{\left[\pi \hat{T}_{w}\left(x_{3}\right)\right]^{3 / 2}} \exp \left(-\frac{|\zeta|^{2}}{\hat{T}_{w}\left(x_{3}\right)}\right),
$$

with an undetermined $\hat{\rho}_{(0)}$.

\section{First order in $\delta$}

The equation and boundary condition for the first order in $\delta$ are given as follows:

$$
\begin{aligned}
\underline{\boldsymbol{\zeta}} \cdot \nabla_{\underline{x}} \hat{f}_{(1)}= & \frac{4}{\sqrt{\pi}} \frac{1}{K} \hat{J}\left(\hat{f}_{(0)}, \hat{f}_{(1)}\right)-\zeta_{3} \partial_{x_{3}} \hat{f}_{(0)}, \\
\hat{f}_{(1)}(\hat{t}, \mathbf{x}, \boldsymbol{\zeta})= & \int_{\zeta * \cdot \mathbf{n}<0} \hat{K}_{B}\left(\hat{T}_{w} ; \zeta, \zeta_{*}\right) \hat{f}_{(1)}\left(\hat{t}, \mathbf{x}, \zeta_{*}\right) d \zeta_{*}, \\
& \text { for } \boldsymbol{\zeta} \cdot \mathbf{n}>0, \underline{\mathbf{x}} \in \partial \mathrm{S} .
\end{aligned}
$$

Equation (33) is an inhomogeneous linear equation with the inhomogeneous term

$$
\begin{aligned}
-\zeta_{3} \partial_{x_{3}} \hat{f}_{(0)} & =-\zeta_{3} \hat{f}_{(0)}\left[\frac{\partial_{x_{3}} \hat{\rho}_{(0)}}{\hat{\rho}_{(0)}}+\frac{\partial_{x_{3}} \hat{T}_{w}}{\hat{T}_{w}}\left(\frac{|\zeta|^{2}}{\hat{T}_{w}}-\frac{3}{2}\right)\right] \\
& =-\zeta_{3} \hat{f}_{(0)}\left[\frac{\partial_{x_{3}} \hat{p}_{(0)}}{\hat{p}_{(0)}}+\frac{\partial_{x_{3}} \hat{T}_{w}}{\hat{T}_{w}}\left(\frac{|\zeta|^{2}}{\hat{T}_{w}}-\frac{5}{2}\right)\right],
\end{aligned}
$$

where $\hat{p}_{(0)}=\hat{\rho}_{(0)} \hat{T}_{w}$ has been used. The corresponding macroscopic quantities are given as

$$
\begin{aligned}
& \hat{\rho}_{(1)}=\int \hat{f}_{(1)} d \boldsymbol{\zeta}, \\
& \hat{\mathbf{v}}_{(1)}=\frac{1}{\hat{\rho}_{(0)}} \int \zeta \hat{f}_{(1)} d \zeta, \\
& \hat{p}_{(1)}=\hat{\rho}_{(0)} \hat{T}_{(1)}+\hat{\rho}_{(1)} \hat{T}_{w}=\frac{2}{3} \int|\zeta|^{2} \hat{f}_{(1)} d \zeta .
\end{aligned}
$$

If we let

$$
\begin{aligned}
& \phi(\hat{t}, \mathbf{x}, \mathbf{c})=\hat{f}_{(1)} / \hat{f}_{(0)}, \\
& \mathbf{c}=\zeta / \hat{T}_{w}^{1 / 2}, \quad \underline{\mathbf{c}}=\left(c_{1}, c_{2}\right),
\end{aligned}
$$

then the above problem is recast as

$$
\begin{aligned}
\underline{\mathbf{c}} \cdot \nabla_{\underline{x}} \phi= & \frac{2}{\sqrt{\pi}} \frac{\hat{\rho}_{(0)}}{K_{*}} \hat{L}_{\hat{T}_{w}}(\phi)-c_{3}\left[\partial_{x_{3}} \ln \hat{p}_{(0)}\right. \\
& \left.+\left(|\mathbf{c}|^{2}-\frac{5}{2}\right) \partial_{x_{3}} \ln \hat{T}_{w}\right],
\end{aligned}
$$

$$
\begin{aligned}
\phi(\hat{t}, \mathbf{x}, \mathbf{c})= & \int_{\mathbf{c}_{*} \cdot \mathbf{n}<0} k_{\hat{T}_{w}}\left(\mathbf{c}, \mathbf{c}_{*}\right) \phi\left(\hat{t}, \mathbf{x}, \mathbf{c}_{*}\right) E\left(\mathbf{c}_{*}\right) d \mathbf{c}_{*}, \\
& \text { for } \mathbf{c} \cdot \mathbf{n}>0, \quad \underline{\mathbf{x}} \in \partial \mathrm{S},
\end{aligned}
$$

where

$$
\begin{aligned}
\hat{L}_{s}(g)= & \int\left[g\left(\mathbf{c}_{*}^{\prime}\right)+g\left(\mathbf{c}^{\prime}\right)-g\left(\mathbf{c}_{*}\right)-g(\mathbf{c})\right] E\left(\mathbf{c}_{*}\right) \\
& \times b_{s}(|\mathbf{e} \cdot \mathbf{C}| / C, C) d \Omega(\mathbf{e}) d \mathbf{c}_{*},
\end{aligned}
$$

$$
\mathbf{c}^{\prime}=\mathbf{c}+(\mathbf{e} \cdot \mathbf{C}) \mathbf{e}, \quad \mathbf{c}_{*}^{\prime}=\mathbf{c}_{*}-(\mathbf{e} \cdot \mathbf{C}) \mathbf{e},
$$

$$
\begin{aligned}
& \mathbf{C}=\mathbf{c}_{*}-\mathbf{c}, \quad C=|\mathbf{C}|, \\
& d \mathbf{c}_{*}=d c_{*_{1}} d c_{*_{2}} d c_{* 3}, \\
& b_{s}(|\mathbf{e} \cdot \mathbf{C}| / C, C)=\hat{B}(|\mathbf{e} \cdot \mathbf{C}| / C, \sqrt{s} C) / \sqrt{s}, \\
& k_{s}\left(\mathbf{c}, \mathbf{c}_{*}\right)=s^{3 / 2} \hat{K}_{B}\left(s ; \sqrt{s} \mathbf{c}, \sqrt{s \mathbf{c}_{*}}\right) / E(\mathbf{c}), \\
& E(\mathbf{c})=\pi^{-3 / 2} \exp \left(-|\mathbf{c}|^{2}\right) .
\end{aligned}
$$

For hard-sphere molecules, $b_{s}=|\mathbf{e} \cdot \mathbf{C}| / 4 \sqrt{2 \pi}$; in the case of

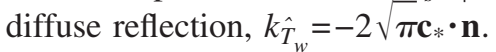

Because of the linearity of the problem and the form of the inhomogeneous term, we can set

$$
\begin{aligned}
\phi(\hat{t}, \mathbf{x}, \mathbf{c})= & \phi_{P}\left(\underline{\mathbf{x}}, \mathbf{c} ; \hat{T}_{w}\left(x_{3}\right), K\left(\hat{t}, x_{3}\right)\right) \partial_{x_{3}} \ln \hat{p}_{(0)} \\
& +\phi_{T}\left(\underline{\mathbf{x}}, \mathbf{c} ; \hat{T}_{w}\left(x_{3}\right), K\left(\hat{t}, x_{3}\right)\right) \partial_{x_{3}} \ln \hat{T}_{w},
\end{aligned}
$$

where $K$ is a kind of local Knudsen number defined by

$$
K=\frac{K_{*}}{\hat{\rho}_{(0)}\left(\hat{t}, x_{3}\right)}=\frac{K_{*} \hat{T}_{w}\left(x_{3}\right)}{\hat{p}_{(0)}\left(\hat{t}, x_{3}\right)} .
$$

Then, $\phi_{P}$ and $\phi_{T}$ are governed by the following equation and boundary condition:

$$
\underline{\mathbf{c}} \cdot \nabla_{\underline{x}} \phi_{\alpha}=\frac{2}{\sqrt{\pi}} \frac{1}{K} \hat{L}_{\hat{T}_{w}}\left(\phi_{\alpha}\right)-\mathrm{I}_{\alpha},
$$

$$
\begin{aligned}
\phi_{\alpha}\left(\underline{\mathbf{x}}, \mathbf{c} ; \hat{T}_{w}, K\right)= & \int_{\mathbf{c}_{*} \cdot \mathbf{n}<0} k_{\hat{T}_{w}}\left(\mathbf{c}, \mathbf{c}_{*}\right) \phi_{\alpha}\left(\underline{\mathbf{x}}, \mathbf{c}_{*} ; \hat{T}_{w}, K\right) E\left(\mathbf{c}_{*}\right) d \mathbf{c}_{*}, \\
& \text { for } \mathbf{c} \cdot \mathbf{n}>0, \underline{\mathbf{x}} \in \partial \mathrm{S},
\end{aligned}
$$

where $\alpha=P$ and $T$, and

$$
\mathrm{I}_{P}=c_{3}, \quad \mathrm{I}_{T}=c_{3}\left(|\mathbf{c}|^{2}-5 / 2\right) .
$$

It is to be noted that $\phi_{P}$ and $\phi_{T}$ depend on $\hat{t}$ and $x_{3}$ only through $\hat{T}_{w}\left(x_{3}\right)$ and $K\left(\hat{t}, x_{3}\right)$. 
By the use of the solutions of the above problems, the first-order solution $\hat{f}_{(1)}$ can be expressed as

$$
\begin{aligned}
\hat{f}_{(1)}= & \hat{f}_{(0)}\left[\phi_{P}\left(\underline{\mathbf{x}}, \frac{\boldsymbol{\zeta}}{\hat{T}_{w}^{1 / 2}} ; \hat{T}_{w}\left(x_{3}\right), K\left(\hat{t}, x_{3}\right)\right) \partial_{x_{3}} \ln \hat{p}_{(0)}\right. \\
& \left.+\phi_{T}\left(\underline{\mathbf{x}}, \frac{\boldsymbol{\zeta}}{\hat{T}_{w}^{1 / 2}} ; \hat{T}_{w}\left(x_{3}\right), K\left(\hat{t}, x_{3}\right)\right) \partial_{x_{3}} \ln \hat{T}_{w}\right] .
\end{aligned}
$$

Since the kernel in the boundary condition $k_{\hat{T}_{w}}$ is locally isotropic (see Sec. II A), it can be shown that the solution of the form

$$
\phi_{\alpha}=c_{3} \Phi_{\alpha}\left(\underline{\mathbf{x}}, \underline{\mathbf{c}}, c_{3}^{2} ; \hat{T}_{w}, K\right)
$$

is compatible with Eq. (43). Then, the macroscopic quantities of the first order given by Eqs. (36a)-(36c) take the following form:

$$
\begin{aligned}
& \hat{\rho}_{(1)}=\hat{T}_{(1)}=\hat{p}_{(1)}=0, \\
& \hat{v}_{3(1)}=\hat{T}_{w}^{1 / 2}\left(u_{P} \partial_{x_{3}} \ln \hat{p}_{(0)}+u_{T} \partial_{x_{3}} \ln \hat{T}_{w}\right), \\
& \hat{v}_{1(1)}=\hat{v}_{2(1)}=0,
\end{aligned}
$$

where

$$
u_{\alpha}\left(\underline{\mathbf{x}} ; \hat{T}_{w}, K\right)=\int c_{3} \phi_{\alpha}\left(\underline{\mathbf{x}}, \mathbf{c} ; \hat{T}_{w}, K\right) E(\mathbf{c}) d \mathbf{c}, \quad(\alpha=P, T) .
$$

Equations (43) and (44) correspond to the well-known problems of Poiseuille flow $(\alpha=P)$ and thermal transpiration $(\alpha=T)$. The former is the flow through a long pipe driven by a (small) uniform pressure gradient, and the latter is that driven by a (small) uniform gradient of the temperature of the pipe wall. Here, we should note that the linearized collision operator $\hat{L}_{\hat{T}_{w}}$ and the scattering kernel $k_{\hat{T}_{w}}$ are parametrized by $\hat{T}_{w}$. If we investigate these two problems starting from the original system (1) and (3), then we are led to the linearized system (43) and (44) with $\hat{T}_{w}=1$. In this sense, Eqs. (43) and (44) are not exactly the same as the equation and boundary condition for the Poiseuille flow and thermal transpiration that have been studied in the literature. We may call these problems the generalized Poiseuille flow and thermal transpiration. However, in the case of hard-sphere molecules and diffuse reflection, since $\hat{L}_{\hat{T}_{w}}$ and $k_{\hat{T}_{w}}$ do not contain $\hat{T}_{w}$, Eqs. (43) and (44) are exactly the equation and boundary condition for these problems.

The Poiseuille flow and thermal transpiration mentioned above have extensively been studied in various situations in the literature (e.g., Refs. 12, 14, and 40-43 for the Poiseuille flow and Refs. 12, 14, and 42-45 for the thermal transpiration). The reader is referred to an exhaustive review by Sharipov and Seleznev ${ }^{46}$ for the works earlier than this article. Concerning the new development, see, for example, Refs. 47-49.

\section{Second order in $\delta$ and diffusion model}

The equation and boundary condition for the second order in $\delta$ are as follows:

$$
\begin{aligned}
\underline{\boldsymbol{\zeta}} \cdot \nabla_{\underline{x}} \hat{f}_{(2)}= & \frac{2}{\sqrt{\pi}} \frac{1}{K_{*}}\left[2 \hat{J}\left(\hat{f}_{(0)}, \hat{f}_{(2)}\right)+\hat{J}\left(\hat{f}_{(1)}, \hat{f}_{(1)}\right)\right] \\
& -\zeta_{3} \partial_{x_{3}} \hat{f}_{(1)}-\partial_{\hat{t}} \hat{f}_{(0)}, \\
\hat{f}_{(2)}(\hat{t}, \mathbf{x}, \boldsymbol{\zeta})= & \int_{\boldsymbol{\zeta}_{*} \cdot \mathbf{n}<0} \hat{K}_{B}\left(\hat{T}_{w} ; \boldsymbol{\zeta}, \boldsymbol{\zeta}_{*}\right) \hat{f}_{(2)}\left(\hat{t}, \mathbf{x}, \boldsymbol{\zeta}_{*}\right) d \boldsymbol{\zeta}_{*}, \\
& \text { for } \boldsymbol{\zeta} \cdot \mathbf{n}>0, \underline{\mathbf{x}} \in \partial \mathrm{S} .
\end{aligned}
$$

Here, we derive the necessary condition for the above boundary-value problem to be solved. We first integrate Eq. (50) over the whole space of $\zeta$ to obtain

$$
\nabla_{\underline{x}} \cdot \int \underline{\boldsymbol{\zeta}} \hat{f}_{(2)} d \zeta+\partial_{t} \hat{\boldsymbol{\rho}}_{(0)}+\partial_{x_{3}}\left(\hat{\boldsymbol{\rho}}_{(0)} \hat{v}_{3(1)}\right)=0 .
$$

Then further integration with respect to $\underline{\mathbf{x}}$ over the cross section $\mathrm{S}$ of the pipe gives

$$
\hat{S} \partial_{t} \hat{\boldsymbol{\rho}}_{(0)}+\partial_{x_{3}}\left(\hat{\boldsymbol{\rho}}_{(0)} \int_{\mathrm{S}} \hat{v}_{3(1)} d \underline{\mathbf{x}}\right)=0
$$

where $\hat{S}=S / D^{2}$ is the area of the cross section $S$ of the pipe in the dimensionless $\left(x_{1}, x_{2}\right)$ plane. In Eq. (53), use has been made of the property

$$
\int \underline{\boldsymbol{\zeta}} \cdot \mathbf{n} \hat{f}_{(2)} d \zeta=0, \quad \text { for } \underline{\mathbf{x}} \in \partial \mathrm{S},
$$

of the boundary condition (51) [cf. Eq. (8)] and the fact that $\hat{\rho}_{(0)}$ does not depend on $\underline{\mathbf{x}}$. With the help of Eq. (48b), Eq. (53) can be rewritten as

$$
\hat{S} \partial_{t} \hat{\rho}_{(0)}+\partial_{x_{3}}\left[\hat{\rho}_{(0)} \hat{T}_{w}^{1 / 2}\left(M_{P} \partial_{x_{3}} \ln \hat{p}_{(0)}+M_{T} \partial_{x_{3}} \ln \hat{T}_{w}\right)\right]=0
$$

where $M_{P}$ and $M_{T}$ are the dimensionless mass-flow rate of the generalized Poiseuille flow and that of the generalized thermal transpiration, respectively [see the paragraph following Eq. (49)], and are defined by

$$
M_{\alpha}\left(\hat{T}_{w}, K ; \mathrm{S}\right)=\int_{\mathrm{S}} u_{\alpha}\left(\underline{\mathbf{x}} ; \hat{T}_{w}, K\right) d \underline{\mathbf{x}}, \quad(\alpha=P, T) .
$$

Since the mass-flow rates depend on the shape of the cross section of the pipe, $\mathrm{S}$ is shown explicitly in $M_{\alpha}$. This is for the convenience in Secs. III and IV. Multiplying Eq. (55) by $\hat{T}_{w}$, which is independent of $\hat{t}$, we obtain the corresponding equation for $\hat{p}_{(0)}$. Since $M_{\alpha}$ depends on the local Knudsen number $K$, and $K$ depends on $\hat{p}_{(0)}$ [Eq. (42)], the equation is written in the following form: 


$$
\begin{aligned}
& \partial_{\hat{t}} \hat{p}_{(0)}+\frac{\hat{T}_{w}}{\hat{S}} \partial_{x_{3}} M=0, \\
& M=\frac{\hat{p}_{(0)}}{\hat{T}_{w}^{1 / 2}}\left[M_{P}\left(\hat{T}_{w}, \frac{\hat{T}_{w} K_{*}}{\hat{p}_{(0)}} ; \mathrm{S}\right) \partial_{x_{3}} \ln \hat{p}_{(0)}\right. \\
& \left.+M_{T}\left(\hat{T}_{w}, \frac{\hat{T}_{w} K_{*}}{\hat{p}_{(0)}} ; \mathrm{S}\right) \partial_{x_{3}} \ln \hat{T}_{w}\right] \text {. }
\end{aligned}
$$

Here, it is noted that if $\tilde{M}$ denotes the dimensional mass-flow rate (per unit time) in the $X_{3}$ direction, then it is related to $M$ as

$$
\tilde{M} / D^{2} \rho_{*}\left(2 R T_{*}\right)^{1 / 2}=M \delta+O\left(\delta^{2}\right) .
$$

Suppose that the mass-flow rates $M_{P}$ and $M_{T}$ are known. Then, since $\hat{T}_{w}$ is a known function of $x_{3}$, Eq. (57a) with Eq. (57b) is the equation for $\hat{p}_{(0)}$ of the diffusion type. If we specify the pressure at the initial time, Eqs. (57a) and (57b) describe the transient pressure distribution and the mass-flow rate along the pipe. However, this does not mean that we are solving the original Boltzmann system, Eqs. (24), (18), and (20), for small $\delta$ for an arbitrary initial distribution $\hat{f}^{0}(\mathbf{x}, \zeta)$. Equation (57) corresponds to Eqs. (24), (18), and (20) for small $\delta$ only when the initial distribution $\hat{f}^{0}$ is a Maxwellian of the form of Eq. (32). Otherwise, the initial distribution is quickly thermalized with the pipe wall in a much shorter time scale and approaches a Maxwellian of the form of Eq. (32). Equation (57) describes the slow time evolution after the thermalization, but not the fast thermalization process itself (the so-called initial layer). Even in this case, however, Eq. (57) is expected to give sufficiently accurate long-time behavior of the solution to the original Boltzmann system. Equation (57) may be applied to a long but finite pipe by specifying the pressures at both ends as the boundary conditions. It is natural to expect that the solution of this problem correctly describes the long-time behavior and the final steady state of the solution to the Boltzmann system when the pipe connects two reservoirs with prescribed pressures. (See also Ref. 39 for the discussion about the initial condition.)

Equation (57) is similar to the generalized Reynolds equation that was first derived for a thin-gap slider bearing ${ }^{50}$ and has been applied to microchannel flows. ${ }^{51-53}$ We should stress, however, that Eq. (57) is applicable to any nonsmall pressure difference and temperature variation. On the other hand, rigorous mathematical derivation of the diffusion equation in the thin-tube limit or thin-gap limit (in the case of a gas between two plates) has been made for a collisionless (or free-molecular) gas. ${ }^{54-57}$ It should also be mentioned that models in the same spirit have been derived and applied in connection with stationary plasma thrusters ${ }^{58-60}$ and that their rigorous mathematical derivation has been worked out in the collisionless case ${ }^{61}$ as well as in the case with a collision term. ${ }^{62}$ Mention should also be made of the fact that an

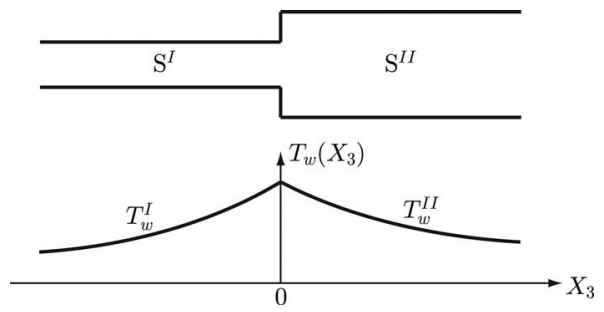

FIG. 1. Connection of two pipes with different cross sections.

extension of Eq. (57) for a binary gas mixture has been made (in the case of a two-dimensional channel) in connection with gas separation. ${ }^{39}$

\section{CONNECTION OF PIPES WITH DIFFERENT CROSS SECTIONS}

In this section, we discuss, on the basis of the diffusion model derived in Sec. II, the case where two long pipes with different cross sections are connected.

\section{A. Connection problem}

Let two long pipes, pipe I $\left(X_{3}<0\right)$ and pipe II $\left(0 \leq X_{3}\right)$, with different cross sections be connected at $X_{3}=0$ (Fig. 1). The cross section $S^{\mathrm{I}}$ (area $S^{\mathrm{I}}$ ) of pipe $\mathrm{I}$ is assumed to be smaller than the cross section $S^{\mathrm{II}}$ (area $S^{\mathrm{II}}$ ) of pipe II, so that $S^{\mathrm{I}} \subset S^{\mathrm{II}}, S^{\mathrm{I}}<S^{\mathrm{II}}$. The temperature of the wall of the whole pipe, denoted by $T_{w}\left(X_{3}\right)\left(-\infty<X_{3}<\infty\right)$ and composed of $T_{w}^{\mathrm{I}}$ $\left(X_{3}<0\right)$ and $T_{w}^{\mathrm{II}}\left(0 \leq X_{3}\right)$, is continuous at the junction $X_{3}$ $=0$, but the gradient may be discontinuous there.

Since the pipes are long, one may expect that the overall behavior of the gas in the connected pipes is described by the diffusion model derived in Sec. II, with a local correction near the junction. We investigate this local correction and derive the connection condition for the diffusion model at the junction.

We nondimensionalize the present problem using the same dimensionless variables as in Sec. II and denote its solution by $F$ instead of $\hat{f}$. We also denote the dimensionless counterparts of $T_{w}^{\mathrm{I}}, T_{w}^{\mathrm{II}}, S^{\mathrm{I}}$, and $S^{\mathrm{II}}$ by $\hat{T}_{w}^{\mathrm{I}}, \hat{T}_{w}^{\mathrm{II}}, \hat{S}^{\mathrm{I}}$, and $\hat{S}^{\mathrm{II}}$, respectively. Then, the dimensionless forms of the equation and boundary conditions for $F$ become as follows. The equation is

$$
\delta^{2} \partial_{\hat{t}} F+\underline{\zeta} \cdot \nabla_{\underline{x}} F+\delta \zeta_{3} \partial_{x_{3}} F=\frac{2}{\sqrt{\pi}} \frac{1}{K *} \hat{J}(F, F) .
$$

The boundary conditions on the walls of pipe I $\left(\underline{\mathbf{x}} \in \partial \mathrm{S}^{\mathrm{I}}, x_{3}\right.$ $<0)$ and pipe II $\left(\underline{\mathbf{x}} \in \partial \mathrm{S}^{\mathrm{II}}, x_{3}>0\right)$, as well as on the wall at the junction $\left(\underline{\mathbf{x}} \in \mathrm{S}^{\mathrm{II}}-\mathrm{S}^{\mathrm{I}}, x_{3}=0\right)$, are

$$
\begin{aligned}
F(\hat{t}, \mathbf{x}, \boldsymbol{\zeta})= & \int_{\zeta * \cdot \mathbf{n}<0} \hat{K}_{B}\left(\hat{T}_{w} ; \zeta, \zeta_{*}\right) F\left(\hat{t}, \mathbf{x}, \zeta_{*}\right) d \zeta_{*}, \\
& \text { for } \boldsymbol{\zeta} \cdot \mathbf{n}>0,
\end{aligned}
$$

where $\mathbf{n}$ is the unit normal vector of the pipe wall and that of the wall at the junction, pointing into the gas $[\mathbf{n}=(0,0,1)$ when $x_{3}=0$ and $\left.\underline{\mathbf{x}} \in \mathrm{S}^{\mathrm{II}}-\mathrm{S}^{\mathrm{I}}\right]$. 
Now let us denote by $F^{\mathrm{I}}$ the restriction of $F$ in pipe I $\left(F^{\mathrm{I}}=F\right.$ for $\left.-\infty<x_{3}<0\right)$ and by $F^{\mathrm{II}}$ that in pipe II $\left(F^{\mathrm{II}}=F\right.$ for $\left.0 \leq x_{3}<\infty\right)$. Then, $F^{\mathrm{I}}$ and $F^{\mathrm{II}}$ satisfy Eq. (59). Further, $F^{\mathrm{I}}$ satisfies the boundary condition (60) on the wall of pipe I, whereas $F^{\mathrm{II}}$ satisfies Eq. (60) on the wall of pipe II as well as on the wall at the junction. In addition, $F^{\mathrm{I}}$ and $F^{\mathrm{II}}$ should be continuous at the junction for the molecular velocity with $\zeta_{3} \neq 0$, i.e.,

$F^{\mathrm{I}}\left(\hat{t}, \underline{\mathbf{x}}, x_{3}=0_{-}, \zeta\right)=F^{\mathrm{II}}\left(\hat{t}, \underline{\mathbf{x}}, x_{3}=0, \zeta\right), \quad$ for $\zeta_{3} \neq 0$ and $\underline{\mathbf{x}} \in \mathrm{S}^{\mathrm{I}}$.

It should be noted that, for the geometry considered here, the velocity distribution function is, in general, discontinuous at $x_{3}=0$ for the molecular velocities with $\zeta_{3}=0$. The perturbation analysis of this problem will be carried out below.

\section{B. Condition at junctions}

Let us first assume that the restriction $F^{J}(J=\mathrm{I}, \mathrm{II})$ is given by the corresponding restriction of the solution $\hat{f}$ of Eqs. (24) and (18) with $T_{w}=T_{w}^{J}$ and $\mathrm{S}$ being $\mathrm{S}^{J}$. If we denote such $F^{J}$ by $F_{f}^{J}$, then, it is expanded in $\delta$ as

$$
F_{f}^{J}=F_{f(0)}^{J}+F_{f(1)}^{J} \delta+F_{f(2)}^{J} \delta^{2}+\cdots, \quad(J=\mathrm{I}, \mathrm{II}),
$$

and, for instance, $F_{f(0)}^{J}$ is given by the following Maxwellians [Eq. (32)]:

$$
\begin{aligned}
& F_{f(0)}^{\mathrm{I}}=\frac{\hat{\boldsymbol{\rho}}_{(0)}^{\mathrm{I}}\left(\hat{t}, x_{3}\right)}{\left[\pi \hat{T}_{w}^{\mathrm{I}}\left(x_{3}\right)\right]^{3 / 2}} \exp \left(-\frac{|\zeta|^{2}}{\hat{T}_{w}^{\mathrm{I}}\left(x_{3}\right)}\right), \\
& F_{f(0)}^{\mathrm{II}}=\frac{\hat{\boldsymbol{\rho}}_{(0)}^{\mathrm{II}}\left(\hat{t}, x_{3}\right)}{\left[\pi \hat{T}_{w}^{\mathrm{II}}\left(x_{3}\right)\right]^{3 / 2}} \exp \left(-\frac{|\zeta|^{2}}{\hat{T}_{w}^{\mathrm{I}}\left(x_{3}\right)}\right),
\end{aligned}
$$

where $\hat{\rho}_{(0)}^{\mathrm{I}}$ and $\hat{\rho}_{(0)}^{\mathrm{II}}$ are undetermined functions.

Concerning the zeroth order of $\delta$, it is easily seen that $F_{f(0)}^{\mathrm{I}}$ and $F_{f(0)}^{\mathrm{II}}$ of Eqs. (63a) and (63b) satisfy the equation and boundary condition for $F^{\mathrm{I}}$ and those for $F^{\mathrm{II}}$, respectively. Therefore, to satisfy the continuity condition (61), we need to impose

$$
\hat{\rho}_{(0)}^{\mathrm{I}}\left(\hat{t}, x_{3}=0_{-}\right)=\hat{\rho}_{(0)}^{\mathrm{II}}\left(\hat{t}, x_{3}=0\right),
$$

or equivalently,

$$
\hat{p}_{(0)}^{\mathrm{I}}\left(\hat{t}, x_{3}=0_{-}\right)=\hat{p}_{(0)}^{\mathrm{II}}\left(\hat{t}, x_{3}=0\right),
$$

where $\hat{p}_{(0)}^{J}=\hat{\rho}_{(0)}^{J} \hat{T}_{w}^{J}$.

At the first order in $\delta, F_{f(1)}^{\mathrm{I}}$ and $F_{f(1)}^{\mathrm{II}}$ are, respectively, the corresponding restriction of $\hat{f}_{(1)}$ with $\hat{T}_{w}=\hat{T}_{w}^{\mathrm{I}}$ and $\mathrm{S}$ being $\mathrm{S}^{\mathrm{I}}$ and that with $\hat{T}_{w}=\hat{T}_{w}^{\mathrm{II}}$ and $\mathrm{S}$ being $\mathrm{S}^{\mathrm{II}}$. However, since $\hat{f}_{(1)}$ has the flow velocity $\hat{v}_{3(1)}$ [Eq. (48b)] in the $x_{3}$ direction, it cannot satisfy the impermeability condition $\left(\hat{v}_{3}=0\right)$ imposed by the boundary condition (60) on the wall at the junction $\left(x_{3}\right.$ $\left.=0, \underline{\mathbf{x}} \in \mathrm{S}^{\mathrm{II}}-\mathrm{S}^{\mathrm{I}}\right)$. Therefore, we seek the restrictions $F^{J}$ of the solution in the following form:

$$
F^{J}=F_{f}^{J}\left(\hat{t}, \underline{\mathbf{x}}, x_{3}, \zeta\right)+G^{J}(\hat{t}, \underline{\mathbf{x}}, z, \zeta),
$$

with

$$
z=x_{3} / \delta
$$

where $G^{J}$ is a correction term with a much shorter length scale of variation $D$ in the $x_{3}$ direction. Note that $G^{\mathrm{I}}$ is defined for $-\infty<z<0$, and $G^{\mathrm{II}}$ for $0 \leq z<\infty$. Corresponding to the form (62), $G^{J}$ is expanded as

$$
G^{J}=G_{(1)}^{J} \delta+G_{(2)}^{J} \delta^{2}+\cdots,
$$

where the expansion is started from the first order, since the zeroth-order solution $F_{f(0)}^{J}$ satisfies the boundary condition. If we substitute Eq. (66) with Eqs. (62) and (68) into the equations and boundary conditions for the restrictions $F^{J}$ and take into account the properties of $F_{f}^{J}$ as well as $G^{J}$, we obtain the equation and boundary conditions for $G_{(1)}^{J}, G_{(2)}^{J}$, etc. The equation for $G_{(1)}^{J}$ thus obtained is as follows:

$$
\underline{\zeta} \cdot \nabla_{\underline{x}} G_{(1)}^{J}+\zeta_{3} \partial_{z} G_{(1)}^{J}=\frac{4}{\sqrt{\pi}} \frac{1}{K_{*}} \hat{J}\left(\left.F_{f(0)}^{J}\right|_{x_{3}=0}, G_{(1)}^{J}\right),
$$

where $\left.F_{f(0)}^{J}\right|_{x_{3}=0}$ indicates $F_{f(0)}^{\mathrm{I}}\left(x_{3}=0_{-}\right)$or $F_{f(0)}^{\mathrm{II}}\left(x_{3}=0\right)$ [see Eq. $(63)]$. The boundary condition for $G_{(1)}^{\mathrm{I}}$ on the wall of pipe I $(z<0)$ is

$$
\begin{aligned}
G_{(1)}^{\mathrm{I}}(\hat{t}, \underline{\mathbf{x}}, z, \boldsymbol{\zeta})= & \int_{\zeta_{*} \cdot \mathbf{n}<0} \hat{K}_{B}\left(\hat{T}_{w}^{\mathrm{I}} ; \boldsymbol{\zeta}, \boldsymbol{\zeta}_{*}\right) G_{(1)}^{\mathrm{I}}\left(\hat{t}, \underline{\mathbf{x}}, z, \boldsymbol{\zeta}_{*}\right) d \boldsymbol{\zeta}_{*}, \\
& \text { for } \boldsymbol{\zeta} \cdot \mathbf{n}>0, \underline{\mathbf{x}} \in \partial \mathrm{S}^{\mathrm{I}} .
\end{aligned}
$$

The boundary condition for $G_{(1)}^{\text {II }}$ on the wall of channel II $(z>0)$ is

$$
\begin{aligned}
G_{(1)}^{\mathrm{II}}(\hat{t}, \underline{\mathbf{x}}, z, \boldsymbol{\zeta})= & \int_{\zeta^{*} \cdot \mathbf{n}<0} \hat{K}_{B}\left(\hat{T}_{w}^{\mathrm{II}} ; \boldsymbol{\zeta}, \boldsymbol{\zeta}_{*}\right) G_{(1)}^{\mathrm{II}}\left(\hat{t}, \underline{\mathbf{x}}, z, \boldsymbol{\zeta}_{*}\right) d \boldsymbol{\zeta}_{*}, \\
& \text { for } \boldsymbol{\zeta} \cdot \mathbf{n}>0, \underline{\mathbf{x}} \in \partial \mathrm{S}^{\mathrm{II}},
\end{aligned}
$$

and that on the wall at the junction $\left(z=0, \underline{\mathbf{x}} \in S^{I I}-S^{I}\right)$ is

$$
\begin{aligned}
G_{(1)}^{\mathrm{II}}(\hat{t}, \underline{\mathbf{x}}, 0, \boldsymbol{\zeta})= & \int_{\zeta * \cdot \mathbf{n}<0} \hat{K}_{B}\left(\hat{T}_{w}^{\mathrm{II}}(0) ; \zeta, \zeta_{*}\right)\left[G_{(1)}^{\mathrm{II}}\left(\hat{t}, \underline{\mathbf{x}}, 0, \boldsymbol{\zeta}_{*}\right)\right. \\
& \left.+F_{f(1)}^{\mathrm{II}}\left(\hat{t}, \underline{\mathbf{x}}, x_{3}=0, \boldsymbol{\zeta}\right)\right] d \boldsymbol{\zeta}_{*}-F_{f(1)}^{\mathrm{II}}\left(\hat{t}, \underline{\mathbf{x}}, x_{3}=0, \boldsymbol{\zeta}\right), \\
& \text { for } \boldsymbol{\zeta} \cdot \mathbf{n}>0, \underline{\mathbf{x}} \in \mathrm{S}^{\mathrm{II}}-\mathrm{S}^{\mathrm{I}} .
\end{aligned}
$$

The continuity condition at the junction $(z=0)$ is given as

$$
\begin{aligned}
G_{(1)}^{\mathrm{I}}\left(\hat{t}, \underline{\mathbf{x}}, 0_{-}, \zeta\right)+F_{f(1)}^{\mathrm{I}}\left(\hat{t}, \underline{\mathbf{x}}, x_{3}=\right. & \left.0_{-}, \zeta\right)=G_{(1)}^{\mathrm{II}}(\hat{t}, \underline{\mathbf{x}}, 0, \zeta) \\
& +F_{f(1)}^{\mathrm{II}}\left(\hat{t}, \underline{\mathbf{x}}, x_{3}=0, \zeta\right), \\
& \text { for } \zeta_{3} \neq 0 \text { and } \underline{\mathbf{x}} \in \mathrm{S}^{\mathrm{I}} .
\end{aligned}
$$

In addition, we need to impose the condition at $z \rightarrow \pm \infty$, i.e.,

$$
\begin{aligned}
& G_{(1)}^{\mathrm{I}} \rightarrow 0 \text { for } z \rightarrow-\infty, \\
& G_{(1)}^{\mathrm{II}} \rightarrow 0 \text { for } z \rightarrow \infty .
\end{aligned}
$$

Here, we assume that the solution of Eqs. (69)-(74) exists and consider a condition satisfied by the solution, from which we deduce the connection condition for $F_{f(1)}^{\mathrm{I}}$ and $F_{f(1)}^{\mathrm{II}}$. 
The integration of Eq. (69) over the whole space of $\zeta$ leads to

$$
\nabla_{\underline{x}} \cdot \int \underline{\zeta} G_{(1)}^{J} d \zeta+\partial_{z} \int \zeta_{3} G_{(1)}^{J} d \zeta=0 .
$$

If we integrate this equation over the cross section $S^{J}$ with respect to $\underline{\mathbf{x}}$ and take into account the fact that the boundary conditions (70) and (71) give $\int \boldsymbol{\zeta} \cdot \mathbf{n} G_{(1)}^{J} d \boldsymbol{\zeta}=0$ (note that $n_{3}$ $=0$ ) on the wall of pipe I and pipe II, we have

$$
\partial_{z} \int_{\mathrm{S}^{J}} \int \zeta_{3} G_{(1)}^{J} d \zeta d \underline{\mathbf{x}}=0 .
$$

Further integration with respect to $z$ from $-\infty$ to 0 for $J=\mathrm{I}$ and from 0 to $\infty$ for $J=\mathrm{II}$, with the help of the conditions at infinity (74), yields

$$
\begin{aligned}
& \int_{S^{\mathrm{I}}} \int \zeta_{3} G_{(1)}^{\mathrm{I}}\left(\hat{t}, \underline{\mathbf{x}}, 0_{-}, \boldsymbol{\zeta}\right) d \zeta d \underline{\mathbf{x}}=0, \\
& \int_{\mathrm{S}^{\mathrm{II}}} \int \zeta_{3} G_{(1)}^{\mathrm{II}}(\hat{t}, \underline{\mathbf{x}}, 0, \boldsymbol{\zeta}) d \zeta d \underline{\mathbf{x}}=0 .
\end{aligned}
$$

Since Eq. (72) gives

$$
\begin{aligned}
& \int \zeta_{3}\left[G_{(1)}^{\mathrm{II}}(\hat{t}, \underline{\mathbf{x}}, 0, \boldsymbol{\zeta})+F_{f(1)}^{\mathrm{II}}\left(\hat{t}, \underline{\mathbf{x}}, x_{3}=0, \zeta\right)\right] d \zeta=0, \\
& \quad \text { for } \underline{\mathbf{x}} \in \mathrm{S}^{\mathrm{II}}-\mathrm{S}^{\mathrm{I}}
\end{aligned}
$$

Eq. (77b) is transformed as

$$
\begin{aligned}
& -\int_{\mathrm{S}^{\mathrm{II}}-\mathrm{S}^{\mathrm{I}}} \int \zeta_{3} F_{f(1)}^{\mathrm{II}}\left(\hat{t}, \underline{\mathbf{x}}, x_{3}=0, \zeta\right) d \zeta d \underline{\mathbf{x}} \\
& \quad+\int_{\mathrm{S}^{\mathrm{I}}} \int \zeta_{3}\left[G_{(1)}^{\mathrm{I}}\left(\hat{t}, \underline{\mathbf{x}}, 0_{-}, \zeta\right)+F_{f(1)}^{\mathrm{I}}\left(\hat{t}, \underline{\mathbf{x}}, x_{3}=0_{-}, \zeta\right)\right] d \zeta d \underline{\mathbf{x}} \\
& \quad-\int_{\mathrm{S}^{\mathrm{I}}} \int \zeta_{3} F_{f(1)}^{\mathrm{II}}\left(\hat{t}, \underline{\mathbf{x}}, x_{3}=0, \zeta\right) d \zeta d \underline{\mathbf{x}}=0,
\end{aligned}
$$

where use has been made of Eq. (73) for the integral over $S^{\mathrm{I}}$. With the help of Eq. (77a), we have

$$
\begin{aligned}
\int_{S^{\mathrm{I}}} \int \zeta_{3} F_{f(1)}^{\mathrm{I}}\left(\hat{t}, \underline{\mathbf{x}}, x_{3}=0_{-}, \zeta\right) d \zeta d \underline{\mathbf{x}} \\
=\int_{\mathrm{S}^{\mathrm{II}}} \int \zeta_{3} F_{f(1)}^{\mathrm{II}}\left(\hat{t}, \underline{\mathbf{x}}, x_{3}=0, \zeta\right) d \zeta d \underline{\mathbf{x}} .
\end{aligned}
$$

We recall that $F_{f(1)}^{\mathrm{I}}$ is the restriction for $x_{3}<0$ of $\hat{f}_{(1)}$ with $\hat{T}_{w}=\hat{T}_{w}^{\mathrm{I}}$ and $\mathrm{S}$ being $\mathrm{S}^{\mathrm{I}}$, and $F_{f(1)}^{\mathrm{II}}$ that for $x_{3} \geq 0$ of $\hat{f}_{(1)}$ with $\hat{T}_{w}=\hat{T}_{w}^{\mathrm{II}}$ and $\mathrm{S}$ being $\mathrm{S}^{\mathrm{II}}$. Therefore, from Eqs. (36b), (48b), and (56), we have

$$
\int_{S^{J}} \int \zeta_{3} F_{f(1)}^{J} d \zeta d \underline{\mathbf{x}}=M^{J}
$$

$$
\begin{aligned}
M^{J}= & \frac{\hat{p}_{(0)}^{J}}{\left(\hat{T}_{w}^{J}\right)^{1 / 2}}\left[M_{P}\left(\hat{T}_{w}^{J}, \frac{\hat{T}_{w}^{J} K_{*}}{\hat{p}_{(0)}^{J}} ; \mathrm{S}^{J}\right) \partial_{x_{3}} \ln \hat{p}_{(0)}^{J}\right. \\
& \left.+M_{T}\left(\hat{T}_{w}^{J}, \frac{\hat{T}_{w}^{J} K_{*}}{\hat{p}_{(0)}^{J}} ; \mathrm{S}^{J}\right) \partial_{x_{3}} \ln \hat{T}_{w}^{J}\right]
\end{aligned}
$$

[see Eq. (57b)]. With this $M^{J}$, Eq. (80) is written as

$$
\left.M^{\mathrm{I}}\right|_{x_{3}=0_{-}}=\left.M^{\mathrm{II}}\right|_{x_{3}=0} .
$$

In summary, the zeroth-order pressures, $\hat{p}_{(0)}^{\mathrm{I}}\left(\hat{t}, x_{3}\right)$ in pipe I and $\hat{p}_{(0)}^{\mathrm{II}}\left(\hat{t}, x_{3}\right)$ in pipe II, are governed by the following equation and connection conditions:

$$
\begin{aligned}
& \partial_{\hat{t}} \hat{p}_{(0)}^{J}+\frac{\hat{T}_{w}^{J}}{\hat{S}^{J}} \partial_{x_{3}} M^{J}=0, \quad(J=\mathrm{I}, \mathrm{II}), \\
& \left.\hat{p}_{(0)}^{\mathrm{I}}\right|_{x_{3}=0_{-}}=\left.\hat{p}_{(0)}^{\mathrm{II}}\right|_{x_{3}=0_{+}}, \\
& \left.M^{\mathrm{I}}\right|_{x_{3}=0_{-}}=\left.M^{\mathrm{II}}\right|_{x_{3}=0_{+}},
\end{aligned}
$$

where $M^{J}$ is defined by Eq. (81b), and $x_{3}=0$ on the righthand sides of Eqs. (65) and (82) have been replaced by $x_{3}$ $=0_{+}$for the later convenience.

\section{Generalization}

Now let us consider the case where pipe I is composed of $N$ straight pipes $\mathrm{I}_{1}, \mathrm{I}_{2}, \ldots, \mathrm{I}_{N}$ with cross sections $\mathrm{S}^{\mathrm{I}_{1}}$, $S^{\mathrm{I}_{2}}, \ldots, S^{\mathrm{I}_{N}}$, whose area in the dimensionless $\left(x_{1}, x_{2}\right)$ plane are denoted by $\hat{S}^{\mathrm{I}_{1}}, \hat{S}^{\mathrm{I}_{2}}, \ldots, \hat{S}^{\mathrm{I}_{N}}$, respectively. The temperature $\hat{T}_{w}^{I_{j}}$ of pipe $\mathrm{I}_{j}(j=1,2, \ldots, N)$ may differ from each other but should be functions of $x_{3}$ only. Let $\hat{p}_{(0)}^{\mathrm{I}_{j}}$ be the leading-order (dimensionless) pressures in pipe $\mathrm{I}_{j}(j=1,2, \ldots, N)$. As is obvious from the analysis in Sec. III B, these pressures are governed by essentially the same equations and connection conditions as in Sec. III B. That is,

$$
\begin{aligned}
& \partial_{\hat{t}} \hat{p}_{(0)}^{J}+\frac{\hat{T}_{w}^{J}}{\hat{S}^{J}} \partial_{x_{3}} M^{J}=0, \quad\left(J=\mathrm{I}_{1}, \mathrm{I}_{2}, \ldots, \mathrm{I}_{N}, \text { and II }\right), \\
& \left.\hat{p}_{(0)}^{\mathrm{I}_{j}}\right|_{x_{3}=0_{-}}=\left.\hat{p}_{(0)}^{\mathrm{II}}\right|_{x_{3}=0_{+}}, \quad(j=1,2, \ldots, N), \\
& \left.\sum_{j=1}^{N} M^{\mathrm{I}}\right|_{x_{3}=0_{-}}=\left.M^{\mathrm{II}}\right|_{x_{3}=0_{+}},
\end{aligned}
$$

where $M^{\mathrm{I}_{j}}$ are defined by

$$
\begin{aligned}
M^{\mathrm{I}_{j}}= & \frac{\hat{p}_{(0)}^{\mathrm{I}_{j}}}{\left(\hat{T}_{w}^{\mathrm{I}_{j}}\right)^{1 / 2}}\left[M_{P}\left(\hat{T}_{w}^{\mathrm{I}_{j}}, \frac{\hat{T}_{w}^{\mathrm{I}_{j} K_{*}}}{\hat{p}_{(0)}^{\mathrm{I}_{j}}} ; \mathrm{S}^{\mathrm{I}_{j}}\right) \partial_{x_{3}} \ln \hat{p}_{(0)}^{\mathrm{I}_{j}}\right. \\
& \left.+M_{T}\left(\hat{T}_{w}^{\mathrm{I}_{j}}, \frac{\hat{T}_{w}^{\mathrm{I}_{j}} K_{*}}{\hat{p}_{(0)}^{\mathrm{I}_{j}}} ; \mathrm{S}^{\mathrm{I}_{j}}\right) \partial_{x_{3}} \ln \hat{T}_{w}^{\mathrm{I}_{j}}\right] .
\end{aligned}
$$




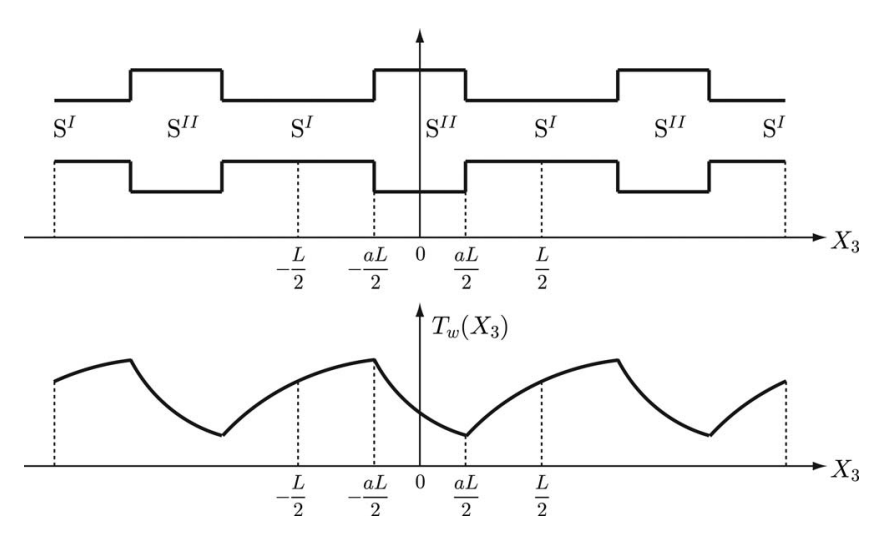

FIG. 2. Schematic figure of the Knudsen compressor.

\section{HOMOGENIZATION: DIFFUSION MODEL FOR THE KNUDSEN COMPRESSOR}

\section{A. Preliminaries}

In this section (Sec. IV), we consider a pipe of periodic structure composed of many basic units of length $L$, as shown in Fig. 2. To be more specific, the part $-L / 2 \leq X_{3}<$ $-a L / 2$ and $a L / 2<X_{3} \leq L / 2$ is a pipe with a smaller cross section $\mathrm{S}^{\mathrm{I}}\left(\operatorname{area} S^{\mathrm{I}}\right)$, and the part $-a L / 2<X_{3}<a L / 2$ is a pipe with a larger cross section $S^{\mathrm{II}}$ [area $S^{\mathrm{II}}\left(>S^{\mathrm{I}}\right)$ ]. We denote by $S$ the cross section of the entire pipe and by $S$ its area, so that $\mathrm{S}$ indicates $\mathrm{S}^{\mathrm{I}}$ or $\mathrm{S}^{\mathrm{II}}$, and $S$ indicates $S^{\mathrm{I}}$ or $S^{\mathrm{II}}$, depending on the position $X_{3}$. The temperature $T_{w}\left(X_{3}\right)$ of the pipe wall is a continuous and periodic function of $X_{3}$ with period $L$. Its gradient may be discontinuous at the junctions $X_{3}$ $=(n \pm a / 2) L(n=0, \pm 1, \pm 2, \ldots)$ of the pipes with different cross sections. We call the part of the pipe with cross section $\mathrm{S}^{\mathrm{I}}$ segment I and that with the cross section $\mathrm{S}^{\mathrm{II}}$ segment II. We assume that the characteristic length of the cross section of each segment is much smaller than its length, so that Eq. (83) and the connection conditions (84) and (85) (and the corresponding result when $S^{I}$ is larger than $S^{I I}$ ) describe the global behavior of the gas. That is,

$$
\partial_{\hat{t}} \hat{p}+\frac{\hat{T}_{w}}{\hat{S}} \partial_{x_{3}} M=0, \quad\left(x_{3} \neq n \pm a / 2 ; n=0, \pm 1, \pm 2, \ldots\right),
$$

$$
\begin{aligned}
M= & \frac{\hat{p}}{\hat{T}_{w}^{1 / 2}}\left[M_{P}\left(\hat{T}_{w}, \frac{\hat{T}_{w} K_{*}}{\hat{p}} ; \mathrm{S}\right) \partial_{x_{3}} \ln \hat{p}\right. \\
& \left.+M_{T}\left(\hat{T}_{w}, \frac{\hat{T}_{w} K_{*}}{\hat{p}} ; \mathrm{S}\right) \partial_{x_{3}} \ln \hat{T}_{w}\right],
\end{aligned}
$$

$\hat{p}$ and $M$ : continuous, $\quad\left(x_{3}=n \pm a / 2\right)$.

Here, the dimensionless variables are the same as in Secs. II and III, but subscript (0) of $\hat{p}$, which indicates the zeroth order in $\delta$, is omitted.

Let us introduce the dimensionless linear density $\Pi$ by

$$
\Pi\left(\hat{t}, x_{3}\right)=\hat{S} \hat{p}\left(\hat{t}, x_{3}\right) / \hat{T}_{w}\left(x_{3}\right) .
$$

$$
\begin{aligned}
& \partial_{\hat{t}} \Pi+\partial_{x_{3}} M=0, \quad\left(x_{3} \neq n \pm a / 2, n=0, \pm 1, \pm 2, \ldots\right), \\
& M=\hat{T}_{w}^{1 / 2} Q_{P}\left(\hat{T}_{w}, \frac{\hat{S}}{\Pi} K_{*} ; \mathrm{S}\right) \partial_{x_{3}} \Pi+\frac{\Pi}{\hat{T}_{w}^{1 / 2}} Q_{T}\left(\hat{T}_{w}, \frac{\hat{S}}{\Pi} K_{*} ; \mathrm{S}\right) \partial_{x_{3}} \hat{T}_{w},
\end{aligned}
$$

$\Pi / \hat{S}$ and $M$ : continuous, $\quad\left(x_{3}=n \pm a / 2\right)$,

where

$$
\begin{aligned}
Q_{P}\left(\hat{T}_{w}, \frac{\hat{S}}{\Pi} K_{*} ; \mathrm{S}\right)= & \frac{1}{\hat{S}} M_{P}\left(\hat{T}_{w}, \frac{\hat{S}}{\Pi} K_{*} ; \mathrm{S}\right), \\
Q_{T}\left(\hat{T}_{w}, \frac{\hat{S}}{\Pi} K_{*} ; \mathrm{S}\right)= & \frac{1}{\hat{S}}\left[M_{P}\left(\hat{T}_{w}, \frac{\hat{S}}{\Pi} K_{*} ; \mathrm{S}\right)\right. \\
& \left.+M_{T}\left(\hat{T}_{w}, \frac{\hat{S}}{\Pi} K_{*} ; \mathrm{S}\right)\right] .
\end{aligned}
$$

Note that the linear density $\Pi$ itself is discontinuous at the junctions.

It should be noted that the diffusion model for the Knudsen pump corresponding to Eqs. (94)-(96) has been constructed for a gas mixture in the case of a two-dimensional channel in Ref. 39, where a new role of the Knudsen pump as a gas separator is pointed out with convincing numerical evidence.

\section{B. Homogenization: Small temperature variation}

Now we try to analyze the flow in the pipe of periodic structure by homogenization. Let the length of the whole pipe $L_{g}$ be much longer than the length of a unit $L$ and define a small parameter $\epsilon$ as $\epsilon=L / L_{g}$. We introduce a new time variable $s$ and a new space coordinate $y$ by

$$
\begin{aligned}
& s=\epsilon^{2} \hat{t}=\left[\left(2 R T_{*}\right)^{1 / 2} D / L_{g}^{2}\right] t, \\
& y=\epsilon x_{3}=X_{3} / L_{g} .
\end{aligned}
$$

For the moment, let us assume that the entire pipe is located in $-L_{g} / 2 \leq X_{3} \leq L_{g} / 2$. Then, the range of $y$ is $-1 / 2 \leq y$ $\leq 1 / 2$. The $x_{3}$ is a variable describing the change in the short scale in each segment, whereas $y$ is a variable corresponding to the change in the long scale throughout the pipe. We assume that $\Pi$ and $M$ are a function of $s, x_{3}$, and $y$, i.e., $\Pi$ $=\Pi_{\epsilon}\left(s, x_{3}, y\right)$ and $M=M_{\epsilon}\left(s, x_{3}, y\right)$, and that they are periodic in $x_{3}$ with period 1 (period $L$ in $X_{3}$ ). Since the wall temperature $T_{w}$ is periodic in $X_{3}$ with period $L$, its dimensionless counterpart $\hat{T}_{w}$ is a function of $x_{3}$ only and periodic with period 1, $\hat{T}_{w}=\hat{T}_{w}\left(x_{3}\right)$. With these new variables, Eqs. (94)-(96) are recast as

$$
\begin{aligned}
\epsilon^{2} \partial_{s} \Pi_{\epsilon}+\epsilon \partial_{y} M_{\epsilon}+\partial_{x_{3}} M_{\epsilon} & =0, \quad(s>0,-1 / 2 \leq y \leq 1 / 2, \\
& \left.-1 / 2 \leq x_{3} \leq 1 / 2, x_{3} \neq \pm a / 2\right),
\end{aligned}
$$

Then, Eqs. (90)-(92) are recast as 


$$
\begin{aligned}
M_{\epsilon}= & \hat{T}_{w}^{1 / 2} Q_{P}\left(\hat{T}_{w}, \frac{\hat{S}}{\Pi_{\epsilon}} K_{*} ; \mathrm{S}\right) \partial_{x_{3}} \Pi_{\epsilon} \\
& +\frac{\Pi_{\epsilon}}{\hat{T}_{w}^{1 / 2}} Q_{T}\left(\hat{T}_{w}, \frac{\hat{S}}{\Pi_{\epsilon}} K_{*} ; \mathrm{S}\right) \partial_{x_{3}} \hat{T}_{w} \\
& +\epsilon \hat{T}_{w}^{1 / 2} Q_{P}\left(\hat{T}_{w}, \frac{\hat{S}}{\Pi_{\epsilon}} K_{*} ; \mathrm{S}\right) \partial_{y} \Pi_{\epsilon},
\end{aligned}
$$

$\Pi_{\epsilon} / \hat{S}$ and $M_{\epsilon}$ : continuous, $\quad\left(x_{3}= \pm a / 2\right)$,

$$
\begin{aligned}
& \Pi_{\epsilon}\left(x_{3}=-1 / 2\right)=\Pi_{\epsilon}\left(x_{3}=1 / 2\right), \\
& M_{\epsilon}\left(x_{3}=-1 / 2\right)=M_{\epsilon}\left(x_{3}=1 / 2\right),
\end{aligned}
$$

where the range of $x_{3}$ is restricted to $[-1 / 2,1 / 2]$ with the periodic condition (102).

In this subsection, we consider the case where the variation of the wall temperature is so small that it is expressed as

$$
\hat{T}_{w}\left(x_{3}\right)=\hat{T}_{w 0}+\hat{T}_{w 1}\left(x_{3}\right) \epsilon,
$$

where $\hat{T}_{w 0}$ is a constant. We seek the solution in the form of expansion in $\epsilon$, that is,

$$
\begin{aligned}
& \Pi_{\epsilon}=\Pi_{(0)}+\Pi_{(1)} \epsilon+\cdots, \\
& M_{\epsilon}=M_{(0)}+M_{(1)} \epsilon+\cdots .
\end{aligned}
$$

In what follows, we denote by the brackets \langle\rangle$,\langle\rangle^{\mathrm{I}}$, and \langle\rangle$^{\mathrm{II}}$ the integrals over the basic segment, the basic segment I, and the basic segment II, respectively, i.e.,

$$
\begin{aligned}
& \langle F\rangle=\langle F\rangle^{\mathrm{I}}+\langle F\rangle^{\mathrm{II}}, \\
& \langle F\rangle^{\mathrm{I}}=\int_{-1 / 2}^{-a / 2} F\left(x_{3}\right) d x_{3}+\int_{a / 2}^{1 / 2} F\left(x_{3}\right) d x_{3}, \\
& \langle F\rangle^{\mathrm{II}}=\int_{-a / 2}^{a / 2} F\left(x_{3}\right) d x_{3} .
\end{aligned}
$$

\section{Zeroth order in $\epsilon$}

Since the variation of $\hat{T}_{w}$ is small, it is likely that the mass flow $M_{\epsilon}$ is also small. Therefore, we assume that $M_{(0)}=0$, which means from Eq. (100) that

$$
\hat{T}_{w 0}^{1 / 2} Q_{P}\left(\hat{T}_{w 0}, \frac{\hat{S}}{\Pi_{(0)}} K_{*} ; \mathrm{S}\right) \partial_{x_{3}} \Pi_{(0)}=0 .
$$

With this assumption, we can perform the analysis without any inconsistency. Equation (106) is the equation for the zeroth order in $\epsilon$. The connection and periodic conditions for $\Pi_{(0)}$ are

$$
\begin{aligned}
& \Pi_{(0)} / \hat{S} \text { : continuous, } \quad\left(x_{3}= \pm a / 2\right), \\
& \Pi_{(0)}\left(x_{3}=-1 / 2\right)=\Pi_{(0)}, \quad\left(x_{3}=1 / 2\right) .
\end{aligned}
$$

Therefore, the solution $\Pi_{(0)}$ is obtained as

$$
\Pi_{(0)}=N(s, y)\left[\hat{S}^{\mathrm{I}}+\left(\hat{S}^{\mathrm{II}}-\hat{S}^{\mathrm{I}}\right) \chi^{\mathrm{II}}\left(x_{3}\right)\right],
$$

where $N(s, y)$ is an unknown (smooth) function of $s$ and $y$, and $\chi^{\mathrm{II}}$ is the characteristic function of the segment II, i.e.,

$$
\chi^{\mathrm{II}}\left(x_{3}\right)= \begin{cases}1, & \left(-\frac{a}{2}<x_{3}<\frac{a}{2}\right), \\ 0, & \text { (otherwise) } .\end{cases}
$$

\section{First order in $\epsilon$}

The equations and associated conditions for the first order in $\epsilon$ become

$$
\begin{aligned}
\partial_{x_{3}} M_{(1)} & =0, \quad\left(x_{3} \neq \pm a / 2\right), \\
M_{(1)}= & \hat{T}_{w 0}^{1 / 2} Q_{P}\left(\hat{T}_{w 0}, \frac{\hat{S}}{\Pi_{(0)}} K_{*} ; \mathrm{S}\right) \partial_{x_{3}} \Pi_{(1)} \\
& +\frac{\Pi_{(0)}}{\hat{T}_{w 0}^{1 / 2}} Q_{T}\left(\hat{T}_{w 0}, \frac{\hat{S}}{\Pi_{(0)}} K_{*} ; \mathrm{S}\right) \partial_{x_{3}} \hat{T}_{w 1} \\
& +\hat{T}_{w 0}^{1 / 2} Q_{P}\left(\hat{T}_{w 0}, \frac{\hat{S}}{\Pi_{(0)}} K_{*} ; \mathrm{S}\right) \partial_{y} \Pi_{(0)},
\end{aligned}
$$

$$
\Pi_{(1)} / \hat{S} \text { and } M_{(1)}: \text { continuous, } \quad\left(x_{3}= \pm a / 2\right),
$$

$$
\begin{aligned}
& \Pi_{(1)}\left(x_{3}=-1 / 2\right)=\Pi_{(1)}\left(x_{3}=1 / 2\right), \\
& M_{(1)}\left(x_{3}=-1 / 2\right)=M_{(1)}\left(x_{3}=1 / 2\right) .
\end{aligned}
$$

From Eq. (111), $M_{(1)}$ is independent of $x_{3}$, i.e., $M_{(1)}$ $=M_{(1)}(s, y)$. Therefore, by integrating Eq. (112) with respect to $x_{3}$ over the basic segment I (i.e., from $-1 / 2$ to $-a / 2$ and from $a / 2$ to $1 / 2$ ), we obtain

$$
\begin{aligned}
(1-a) M_{(1)}= & \hat{T}_{w 0}^{1 / 2} Q_{P}^{\mathrm{I}}\left(\Pi_{(1)-}^{\mathrm{I}}-\Pi_{(1)+}^{\mathrm{I}}\right) \\
& +\frac{\hat{S}^{\mathrm{I}} N}{\hat{T}_{w 0}^{1 / 2}} Q_{T}^{\mathrm{I}}\left[\hat{T}_{w 1}\left(-\frac{a}{2}\right)-\hat{T}_{w 1}\left(\frac{a}{2}\right)\right] \\
& +\hat{S}^{\mathrm{I}}(1-a) \hat{T}_{w 0}^{1 / 2} Q_{P}^{\mathrm{I}} \partial_{y} N,
\end{aligned}
$$

where

$$
\begin{aligned}
& Q_{P}^{\mathrm{I}}=Q_{P}\left(\hat{T}_{w 0}, K_{*} / N ; \mathrm{S}^{\mathrm{I}}\right), \\
& Q_{T}^{\mathrm{I}}=Q_{T}\left(\hat{T}_{w 0}, K_{*} / N ; \mathrm{S}^{\mathrm{I}}\right), \\
& \Pi_{(1)-}^{\mathrm{I}}=\Pi_{(1)}\left(s,(-a / 2)_{-}, y\right), \\
& \Pi_{(1)+}^{\mathrm{I}}=\Pi_{(1)}\left(s,(a / 2)_{+}, y\right) .
\end{aligned}
$$

Similarly, by integrating Eq. (112) with respect to $x_{3}$ over the basic segment II (i.e., from $-a / 2$ to $a / 2$ ), we have 


$$
\begin{aligned}
a M_{(1)}= & \hat{T}_{w 0}^{1 / 2} Q_{P}^{\mathrm{II}}\left(\Pi_{(1)+}^{\mathrm{II}}-\Pi_{(1)-}^{\mathrm{II}}\right)+\frac{\hat{S}^{\mathrm{II}} N}{\hat{T}_{w 0}^{1 / 2}} Q_{T}^{\mathrm{II}}\left[\hat{T}_{w 1}\left(\frac{a}{2}\right)\right. \\
& \left.-\hat{T}_{w 1}\left(-\frac{a}{2}\right)\right]+\hat{S}^{\mathrm{II}} a \hat{T}_{w 0}^{1 / 2} Q_{P}^{\mathrm{II}} \partial_{y} N,
\end{aligned}
$$

where

$$
\begin{aligned}
& Q_{P}^{\mathrm{II}}=Q_{P}\left(\hat{T}_{w 0}, K_{*} / N ; \mathrm{S}^{\mathrm{II}}\right), \\
& Q_{T}^{\mathrm{II}}=Q_{T}\left(\hat{T}_{w 0}, K_{*} / N ; \mathrm{S}^{\mathrm{II}}\right), \\
& \Pi_{(1)-}^{\mathrm{II}}=\Pi_{(1)}\left(s,(-a / 2)_{+}, y\right), \\
& \Pi_{(1)+}^{\mathrm{II}}=\Pi_{(1)}\left(s,(a / 2)_{-}, y\right) .
\end{aligned}
$$

Since $\left(\Pi_{(1)+}^{\mathrm{I}}-\Pi_{(1)-}^{\mathrm{I}}\right) / \hat{S}^{\mathrm{I}}=\left(\Pi_{(1)_{+}}^{\mathrm{II}}-\Pi_{(1)-}^{\mathrm{II}}\right) / \hat{S}^{\mathrm{II}}$ holds because of Eq. (113), we can eliminate $\Pi_{(1)_{+}}^{\mathrm{I}}, \Pi_{(1)-}^{\mathrm{I}}, \Pi_{(1)+}^{\mathrm{II}}$, and $\Pi_{(1)-}^{\mathrm{II}}$ from Eqs. (115) and (117) to obtain the expression of $M_{(1)}(s, y)$ in terms of $N(s, y)$, i.e.,

$$
\begin{aligned}
M_{(1)}(s, y)= & \hat{T}_{w 0}^{1 / 2}\left(\frac{a}{\hat{S}^{\mathrm{II}} Q_{P}^{\mathrm{II}}}+\frac{1-a}{\hat{S}^{\mathrm{I}} Q_{P}^{\mathrm{I}}}\right)^{-1}\left\{\partial_{y} N+\frac{N}{\hat{T}_{w 0}}\right. \\
& \left.\times\left(\frac{Q_{T}^{\mathrm{II}}}{Q_{P}^{\mathrm{II}}}-\frac{Q_{T}^{\mathrm{I}}}{Q_{P}^{\mathrm{I}}}\right)\left[\hat{T}_{w 1}\left(\frac{a}{2}\right)-\hat{T}_{w 1}\left(-\frac{a}{2}\right)\right]\right\},
\end{aligned}
$$

or using the original $M_{P}$ and $M_{T}$,

$$
\begin{aligned}
M_{(1)}(s, y)= & \hat{T}_{w 0}^{1 / 2}\left(\frac{a}{M_{P}^{\mathrm{II}}}+\frac{1-a}{M_{P}^{\mathrm{I}}}\right)^{-1}\left\{\partial_{y} N+\frac{N}{\hat{T}_{w 0}}\right. \\
& \left.\times\left(\frac{M_{T}^{\mathrm{II}}}{M_{P}^{\mathrm{II}}}-\frac{M_{T}^{\mathrm{I}}}{M_{P}^{\mathrm{I}}}\right)\left[\hat{T}_{w 1}\left(\frac{a}{2}\right)-\hat{T}_{w 1}\left(-\frac{a}{2}\right)\right]\right\},
\end{aligned}
$$

where

$$
\begin{aligned}
& M_{P}^{\mathrm{I}}=M_{P}\left(\hat{T}_{w 0}, K_{*} / N ; \mathrm{S}^{\mathrm{I}}\right), \\
& M_{T}^{\mathrm{I}}=M_{T}\left(\hat{T}_{w 0}, K_{*} / N ; \mathrm{S}^{\mathrm{I}}\right), \\
& M_{P}^{\mathrm{II}}=M_{P}\left(\hat{T}_{w 0}, K_{*} / N ; \mathrm{S}^{\mathrm{II}}\right), \\
& M_{T}^{\mathrm{II}}=M_{T}\left(\hat{T}_{w 0}, K_{*} / N ; \mathrm{S}^{\mathrm{II}}\right) .
\end{aligned}
$$

\section{Second order in $\epsilon$ and diffusion model}

If we proceed to the second order in $\epsilon$, we have the following equation and associated conditions:

$$
\begin{aligned}
& \partial_{s} \Pi_{(0)}+\partial_{y} M_{(1)}+\partial_{x_{3}} M_{(2)}=0,\left(x_{3} \neq \pm a / 2\right), \\
& \Pi_{(2)} / \hat{S} \text { and } M_{(2)}: \text { continuous, } \quad\left(x_{3}= \pm a / 2\right), \\
& \Pi_{(2)}\left(x_{3}=-1 / 2\right)=\Pi_{(2)}\left(x_{3}=1 / 2\right), \\
& M_{(2)}\left(x_{3}=-1 / 2\right)=M_{(2)}\left(x_{3}=1 / 2\right),
\end{aligned}
$$

where we omit the explicit expression of $M_{(2)}$ for simplicity.
With the help of the periodicity and continuity of $M_{(2)}$, the integration of Eq. (122) with respect to $x_{3}$ over the basic unit (i.e., from $-1 / 2$ to $1 / 2$ ) yields

$$
\partial_{s}\left\langle\Pi_{(0)}\right\rangle+\partial_{y}\left\langle M_{(1)}\right\rangle=0 .
$$

Since $M_{(1)}$ is constant in $x_{3}$, we have $\left\langle M_{(1)}\right\rangle=M_{(1)}$. In addition, Eq. (109) gives

$$
\begin{aligned}
\left\langle\Pi_{(0)}\right\rangle & =\left\langle\hat{S}^{\mathrm{I}}+\left(\hat{S}^{\mathrm{II}}-\hat{S}^{\mathrm{I}}\right) \chi^{\mathrm{II}}\left(x_{3}\right)\right\rangle N(s, y) \\
& =\left[\hat{S}^{\mathrm{I}}(1-a)+\hat{S}^{\mathrm{II}} a\right] N(s, y) .
\end{aligned}
$$

Therefore, Eq. (125) is written as

$$
\left[\hat{S}^{\mathrm{I}}(1-a)+\hat{S}^{\mathrm{II}} a\right] \partial_{s} N(s, y)+\partial_{y} M_{(1)}=0 .
$$

Equations (127) and (119) [or Eq. (120)], together with appropriate initial and boundary conditions, form a system of diffusion type that determines $N(s, y)$. From Eq. (93), the pressure $\hat{p}_{\epsilon}$ corresponding to $\Pi_{\epsilon}$ is written as

$$
p_{\epsilon}\left(s, x_{3}, y\right)=\Pi_{\epsilon} \hat{T}_{w} / \hat{S}=\Pi_{(0)} \hat{T}_{w 0} / \hat{S}+O(\epsilon) .
$$

Therefore, the zeroth-order pressure $\hat{p}_{(0)}\left(s, x_{3}, y\right)$, averaged over one period, is given by

$$
\left\langle\hat{p}_{(0)}\right\rangle(s, y)=\left\langle\Pi_{(0)}\right\rangle^{\mathrm{I}} \hat{T}_{w 0} / \hat{S}^{\mathrm{I}}+\left\langle\Pi_{(0)}\right\rangle \mathrm{I} \hat{T}_{w 0} / \hat{S}^{\mathrm{II}}=N(s, y) \hat{T}_{w 0} .
$$

\section{Homogenization: Large temperature variation}

In this section, we investigate the case where the temperature variation is large. For this purpose, we follow the method used in Ref. 35. First, we introduce a new time variable $\tau$ that is different from $s$ in Eq. (98a) as

$$
\tau=\epsilon \hat{t}=\frac{\left(2 R T_{*}\right)^{1 / 2} D}{L L_{g}} t,
$$

and assume that $\Pi$ and $M$ are the functions of $\tau, x_{3}$, and $y$, i.e., $\Pi=\Pi_{\epsilon}\left(\tau, x_{3}, y\right)$ and $M=M_{\epsilon}\left(\tau, x_{3}, y\right)$. Then, we have, in place of Eq. (99), the following equation:

$$
\begin{aligned}
\epsilon \partial_{\tau} \Pi_{\epsilon}+\epsilon \partial_{y} M_{\epsilon}+\partial_{x_{3}} M_{\epsilon}= & 0, \quad(\tau>0,-1 / 2 \leq y \leq 1 / 2, \\
& \left.-1 / 2 \leq x_{3} \leq 1 / 2, x_{3} \neq \pm a / 2\right) .
\end{aligned}
$$

The other equations, Eqs. (100) and (101), are unchanged (except that $s$ is replaced by $\tau$ ). As before, the functions $\Pi_{\epsilon}$ and $M_{\epsilon}$ are assumed to be periodic with period 1 in $x_{3}$ and expanded as Eq. (104).

\section{Zeroth order in $\epsilon$}

If we pick up the zeroth-order terms, we have the following equations and conditions:

$$
\begin{aligned}
& \partial_{x_{3}} M_{(0)}=0, \quad\left(x_{3} \neq \pm a / 2\right), \\
& M_{(0)}=\hat{T}_{w}^{1 / 2} Q_{P(0)} \partial_{x_{3}} \Pi_{(0)}+\Pi_{(0)} \hat{T}_{w}^{-1 / 2} Q_{T(0)} \partial_{x_{3}} \hat{T}_{w},
\end{aligned}
$$




$$
\begin{aligned}
& \Pi_{(0)} / \hat{S} \text { and } M_{(0)}: \text { continuous, } \quad\left(x_{3}= \pm a / 2\right), \\
& \Pi_{(0)}\left(x_{3}=-1 / 2\right)=\Pi_{(0)}\left(x_{3}=1 / 2\right), \\
& M_{(0)}\left(x_{3}=-1 / 2\right)=M_{(0)}\left(x_{3}=1 / 2\right),
\end{aligned}
$$

where

$$
\begin{aligned}
Q_{P(0)} & =Q_{P}\left(\hat{T}_{w}, \frac{\hat{S}}{\Pi_{(0)}} K_{*} ; \mathrm{S}\right), \\
Q_{T(0)} & =Q_{T}\left(\hat{T}_{w}, \frac{\hat{S}}{\Pi_{(0)}} K_{*} ; \mathrm{S}\right) .
\end{aligned}
$$

From Eq. (132), $M_{(0)}$ does not depend on $x_{3}$, i.e., $M_{(0)}$ $=M_{(0)}(\tau, y)$. Let us put

$$
\begin{aligned}
& \Pi_{(0)}\left(\tau, x_{3}, y\right)=N(\tau, y) \Pi_{\#}\left(\tau, x_{3}, y\right), \\
& M_{(0)}(\tau, y)=N(\tau, y) M_{\#}(\tau, y),
\end{aligned}
$$

with

$$
N(\tau, y)=\left\langle\Pi_{(0)}\right\rangle, \quad\left\langle\Pi_{\#}\right\rangle=1 .
$$

Then, Eqs. (133)-(135) are recast as

$$
\begin{aligned}
& M_{\#}=\hat{T}_{w}^{1 / 2} Q_{P \#} \partial_{x_{3}} \Pi_{\#}+\frac{\Pi_{\#}}{\hat{T}_{w}^{1 / 2}} Q_{T \#} \partial_{x_{3}} \hat{T}_{w}, \\
& \Pi_{\#} / \hat{S}: \text { continuous, } \quad\left(x_{3}= \pm a / 2\right), \\
& \Pi_{\#}\left(x_{3}=-1 / 2\right)=\Pi_{\#}\left(x_{3}=1 / 2\right),
\end{aligned}
$$

where

$$
\begin{aligned}
Q_{P \#} & =Q_{P}\left(\hat{T}_{w}, \frac{\hat{S}}{\Pi_{\#}} \frac{K_{*}}{N} ; \mathrm{S}\right), \\
Q_{T \#} & =Q_{T}\left(\hat{T}_{w}, \frac{\hat{S}}{\Pi_{\#}} \frac{K_{*}}{N} ; \mathrm{S}\right) .
\end{aligned}
$$

Equation (139) shows that the dependence of $M_{\#}$ and of $\Pi_{\#}$ on $\tau$ and $y$ is through $N$. Therefore, the solution $\left(\Pi_{\#}, M_{\#}\right)$ of Eqs. (139)-(141) is obtained in the following form:

$$
\Pi_{\#}=\Pi_{\#}\left(x_{3}, K_{*} / N\right), \quad M_{\#}=M_{\#}\left(K_{*} / N\right) .
$$

In Ref. 35, the equation corresponding to Eq. (131) is linearized under the assumption that $\Pi_{\epsilon}$ in $Q_{P}$ and $Q_{T}$ in Eq. (100) are replaced by a reference linear density. In addition, the functions corresponding to $Q_{P}$ and $Q_{T}$ are explicit because of the simplified collision model. Thus, the existence of the solution corresponding to $\Pi_{\#}$ and $M_{\#}$ can be established. In contrast, in the present case, we assume that the solution $\left(\Pi_{\#}, M_{\#}\right)$ exists. It is verified by the numerical analysis.

\section{Higher order in $\epsilon$ and the diffusion model}

Following Ref. 35, instead of carrying out a straightforward expansion, we interpret the expansions (104a) and (104b) in a different way. That is, we assume that the coefficients $\Pi_{(0)}, \Pi_{(1)}, \ldots$ and $M_{(0)}, M_{(1)}, \ldots$, which are of the quantities of $O(1)$, also depend on $\epsilon$, and we impose the normalization condition

$$
\left\langle\Pi_{(1)}\right\rangle=\left\langle\Pi_{(2)}\right\rangle=\cdots=0 .
$$

A remark about this will be given in Sec. IV C 3.

Now we can write Eq. (131) with Eq. (132) in the following form:

$$
\begin{aligned}
& \partial_{\tau} \Pi_{(0)}+\partial_{y} M_{(0)}+\partial_{x_{3}} M_{(1)}+\epsilon\left(\partial_{\tau} \Pi_{(1)}+\partial_{y} M_{(1)}+\partial_{x_{3}} M_{(2)}\right) \\
& \quad=O\left(\epsilon^{2}\right), \quad\left(x_{3} \neq \pm a / 2\right),
\end{aligned}
$$

$$
\begin{aligned}
M_{(1)}= & \hat{T}_{w}^{1 / 2} Q_{P(0)} \partial_{x_{3}} \Pi_{(1)}+\frac{\Pi_{(1)}}{\hat{T}_{w}^{1 / 2}}\left\{\left[Q_{T(0)}-\frac{\hat{S} K_{*}}{\Pi_{(0)}} \partial_{2} Q_{T(0)}\right]\right. \\
& \left.\times \partial_{x_{3}} \hat{T}_{w}-\hat{T}_{w} \frac{\hat{S} K_{*}}{\Pi_{(0)}^{2}} \partial_{2} Q_{P(0)} \partial_{x_{3}} \Pi_{(0)}\right\}+\hat{T}_{w}^{1 / 2} Q_{P(0)} \partial_{y} \Pi_{(0)},
\end{aligned}
$$

$$
M_{(2)}=\cdots,
$$

$$
\begin{aligned}
& {\left[\Pi_{(1)}+\Pi_{(2)} \epsilon+O\left(\epsilon^{2}\right)\right] / \hat{S},} \\
& M_{(1)}+M_{(2)} \epsilon+O\left(\epsilon^{2}\right),
\end{aligned}
$$

$$
\left[\Pi_{(1)}+\Pi_{(2)} \epsilon+O\left(\epsilon^{2}\right)\right]_{x_{3}=-1 / 2}=\left[\Pi_{(1)}+\Pi_{(2)} \epsilon+O\left(\epsilon^{2}\right)\right]_{x_{3}=1 / 2},
$$

$$
\left[M_{(1)}+M_{(2)} \epsilon+O\left(\epsilon^{2}\right)\right]_{x_{3}=-1 / 2}=\left[M_{(1)}+M_{(2)} \epsilon+O\left(\epsilon^{2}\right)\right]_{x_{3}=1 / 2},
$$

where $\partial_{2} Q_{P, T(0)}=\partial_{z_{2}} Q_{P, T}\left(z_{1}, z_{2} ; \mathrm{S}\right)$ at $z_{1}=\hat{T}_{w}$ and $z_{2}=\hat{S} K_{*}$ $/ \Pi_{(0)}$. Integration of Eq. (145) with respect to $x_{3}$ over the basic segment $\left(-1 / 2<x_{3}<1 / 2\right)$ leads to

$$
\partial_{\tau}\left\langle\Pi_{(0)}\right\rangle+\partial_{y} M_{(0)}+\epsilon \partial_{y}\left\langle M_{(1)}\right\rangle=O\left(\epsilon^{2}\right) .
$$

Subtracting $\partial_{\tau}\left\langle\Pi_{(0)}\right\rangle$ and $\partial_{y} M_{(0)}$, which are of the order of unity individually, from the $\epsilon^{0}$-order part of Eq. (145) and add the sum, which is of the order of $\epsilon$ and is equal to $-\epsilon \partial_{y}\left\langle M_{(1)}\right\rangle+O\left(\epsilon^{2}\right)$ [see Eq. (150)], to the $\epsilon$-order part of the same equation, we obtain the following equation:

$$
\begin{gathered}
\partial_{\tau}\left(\Pi_{(0)}-\left\langle\Pi_{(0)}\right\rangle\right)+\partial_{x_{3}} M_{(1)}+\epsilon\left[-\partial_{y}\left\langle M_{(1)}\right\rangle+\partial_{\tau} \Pi_{(1)}+\partial_{y} M_{(1)}\right. \\
\left.\quad+\partial_{x_{3}} M_{(2)}\right]=O\left(\epsilon^{2}\right), \quad\left(x_{3} \neq \pm a / 2\right) .
\end{gathered}
$$

If we consider the leading-order terms, Eq. (151) and the conditions (148) and (149) are reduced to

$$
\begin{aligned}
& \partial_{x_{3}} M_{(1)}=-\partial_{\tau}\left(\Pi_{(0)}-\left\langle\Pi_{(0)}\right\rangle\right), \quad\left(x_{3} \neq \pm a / 2\right), \\
& \Pi_{(1)} / \hat{S} \text { and } M_{(1)}: \text { continuous, } \quad\left(x_{3}= \pm a / 2\right),
\end{aligned}
$$




$$
\begin{aligned}
& \Pi_{(1)}\left(x_{3}=-1 / 2\right)=\Pi_{(1)}\left(x_{3}=1 / 2\right), \\
& M_{(1)}\left(x_{3}=-1 / 2\right)=M_{(1)}\left(x_{3}=1 / 2\right) .
\end{aligned}
$$

Let us consider the system composed of Eqs. (152), (146), (153), and (154). Equations (152) and (146) are recast in the following form by the use of Eqs. (137), (138), and (143),

$$
\begin{gathered}
\partial_{x_{3}} M_{(1)}=-\left(\Pi_{\#}-1-\frac{K_{*}}{N} \partial_{2} \Pi_{\#}\right) \partial_{\tau} N, \quad\left(x_{3} \neq \pm a / 2\right), \\
M_{(1)}-\hat{T}_{w}^{1 / 2} Q_{P \#} \partial_{x_{3}} \Pi_{(1)}-\frac{\Pi_{(1)}}{\hat{T}_{w}^{1 / 2}}\left\{\left[Q_{T \#}-\frac{\hat{S}}{\Pi_{\#}} \frac{K_{*}}{N} \partial_{2} Q_{T \#}\right]\right. \\
\left.\times \partial_{x_{3}} \hat{T}_{w}-\hat{T}_{w} \frac{\hat{S}}{\Pi_{\#}^{2}} \frac{K_{*}}{N} \partial_{2} Q_{P \#} \partial_{x_{3}} \Pi_{\#}\right\} \\
=\hat{T}_{w}^{1 / 2} Q_{P \#}\left(\Pi_{\#}-\frac{K_{*}}{N} \partial_{2} \Pi_{\#}\right) \partial_{y} N,
\end{gathered}
$$

where $\partial_{2} \Pi_{\#}=\partial_{z} \Pi_{\#}\left(x_{3}, z\right) \quad$ at $\quad z=K_{*} / N$, and $\partial_{2} Q_{P, T \#}$ $=\partial_{z_{2}} Q_{P, T}\left(z_{1}, z_{2} ; \mathrm{S}\right)$ at $z_{1}=\hat{T}_{w}$ and $z_{2}=\hat{S} K_{*} /\left(\Pi_{\#} N\right)$. Equations (155) and (156) form the inhomogeneous linear system for $\Pi_{(1)}$ and $M_{(1)}$, where the right-hand sides are the inhomogeneous terms. As is easily seen from Eq. (152), the right-hand side of Eq. (155) satisfies the compatibility condition $\langle\cdot\rangle=0$. Therefore, this system is solvable. We try to obtain an approximate solution allowing the error of the order of $\epsilon$. With the help of Eqs. (137), (138), (143), and (150), we can rewrite Eq. (155) in the following form:

$$
\begin{aligned}
\partial_{x_{3}} M_{(1)}= & \left(\Pi_{\#}-1-\frac{K_{*}}{N} \partial_{2} \Pi_{\#}\right)\left(M_{\#}-\frac{K_{*}}{N} M_{\#}^{\prime}\right) \partial_{y} N \\
& +O(\epsilon), \quad\left(x_{3} \neq \pm a / 2\right) .
\end{aligned}
$$

Let us denote by $\left(\Pi_{(1)}^{a}, M_{(1)}^{a}\right)$ the approximate solution, i.e., the solution of Eq. (157) with the $O(\epsilon)$ term neglected and Eqs. (156), (153), and (154). Then, $\left(\Pi_{(1)}^{a}, M_{(1)}^{a}\right)$ is expressed in the following form:

$$
\begin{aligned}
& \Pi_{(1)}^{a}\left(\tau, x_{3}, y\right)=\Pi_{\dot{\dagger}}\left(x_{3}, \frac{K_{*}}{N}\right) \partial_{y} N, \\
& M_{(1)}^{a}\left(\tau, x_{3}, y\right)=M_{\dagger}\left(x_{3}, \frac{K_{*}}{N}\right) \partial_{y} N,
\end{aligned}
$$

with

$$
\begin{aligned}
& \Pi_{+}\left(x_{3}, \frac{K_{*}}{N}\right)=\Pi_{S(1)}\left(x_{3}, \frac{K_{*}}{N}\right)-\Pi_{H(1)}\left(x_{3}, \frac{K_{*}}{N}\right) \frac{\left\langle\Pi_{S(1)}\right\rangle}{\left\langle\Pi_{H(1)}\right\rangle}, \\
& M_{+}\left(x_{3}, \frac{K_{*}}{N}\right)=M_{S(1)}\left(x_{3}, \frac{K_{*}}{N}\right)-M_{H(1)}\left(x_{3}, \frac{K_{*}}{N}\right) \frac{\left\langle\Pi_{S(1)}\right\rangle}{\left\langle\Pi_{H(1)}\right\rangle} .
\end{aligned}
$$

Here, $\left(\Pi_{H(1)}, M_{H(1)}\right)$ and $\left(\Pi_{S(1)}, M_{S(1)}\right)$ are the general solution of the homogeneous system and a particular solution of the inhomogeneous system (with $\partial_{y} N=1$ ), respectively, and
$\left\langle\Pi_{H(1)}\right\rangle \neq 0$ is assumed. The $\Pi_{\dagger}$ satisfies the normalization condition $\left\langle\Pi_{\dagger}\right\rangle=0$. As a consequence, the solution $\left(\Pi_{(1)}\right.$, $\left.M_{(1)}\right)$ is given as

$$
\begin{aligned}
& \Pi_{(1)}\left(\tau, x_{3}, y\right)=\Pi_{\dagger}\left(x_{3}, \frac{K_{*}}{N}\right) \partial_{y} N+O(\epsilon), \\
& M_{(1)}\left(\tau, x_{3}, y\right)=M_{\dot{\dagger}}\left(x_{3}, \frac{K_{*}}{N}\right) \partial_{y} N+O(\epsilon) .
\end{aligned}
$$

If we use Eqs. (137), (138), (143), and (160b) in Eq. (150) and neglect the terms of $O\left(\epsilon^{2}\right)$, we have

$$
\begin{aligned}
& \partial_{\tau} N+\partial_{y} \mathcal{M}=0, \\
& \mathcal{M}=N M_{\#}\left(\frac{K_{*}}{N}\right)+\epsilon\left\langle M_{\dagger}\left(x_{3}, \frac{K_{*}}{N}\right)\right\rangle \partial_{y} N,
\end{aligned}
$$

where $\mathcal{M}=M_{(0)}+\epsilon\left\langle M_{(1)}\right\rangle+O\left(\epsilon^{2}\right)$. This is the equation for $N(\tau, y)$, which corresponds to the result of Ref. 35 .

If we write the pressure $\hat{p}_{\epsilon}$ corresponding to $\Pi_{\epsilon}$ in the form of expansion

$$
\hat{p}_{\epsilon}=\hat{p}_{(0)}+\hat{p}_{(1)} \epsilon+\cdots,
$$

then, from Eqs. (93), (137), and (143), the zeroth-order pressure $\hat{p}_{(0)}$ is given as

$$
\hat{p}_{(0)}\left(\tau, x_{3}, y\right)=\Pi_{(0)} \hat{T}_{w} / \hat{S}=N(\tau, y) P_{\#}\left(x_{3}, K_{*} / N\right),
$$

where

$$
P_{\#}\left(x_{3}, K * / N\right)=\Pi_{\#}\left(x_{3}, K * / N\right) \hat{T}_{w} / \hat{S} .
$$

\section{Solution procedure and remarks}

To summarize, the numerical solution procedure is as follows. For many specified values of $K_{*} / N$, we solve the nonlinear ordinary differential equation (139) with the conditions (140) and (141) numerically to construct a database for $\Pi_{\#}\left(x_{3}, K_{*} / N\right)$ and $M_{\#}\left(K_{*} / N\right)$. Then, using this database, we solve the system, Eqs. (157) (with $\partial_{y} N=1$ and $\epsilon$-order term neglected), (156) (with $\left.\partial_{y} N=1\right),(153)$, and (154), numerically to build a database of the normalized solution $\left(\Pi_{\dagger}, M_{\dagger}\right)$. Finally, with the help of the databases, we solve Eq. (161), supplemented with appropriate initial and boundary conditions, to obtain $N(\tau, y)$. This $N$, together with $\Pi_{\#}$ and $M_{\#}$, gives the leading-order quantities $\Pi_{(0)}, M_{(0)}$, and $\hat{p}_{(0)}$ in the form of Eqs. (137) and (163).

Finally, we remark on the diffusion equation (161). In the case of small temperature variation in Sec. IV B, we were able to derive a diffusion model (127) by a straightforward expansion in $\epsilon$ of the Hilbert-type. ${ }^{5,63,64}$ If we carry out the corresponding expansion in the case of large temperature variation in Sec. IV C, we cannot obtain a diffusion equation. This is the reason why a different type of expansion, which is of the Chapman-Enskog-type ${ }^{5,63,64}$ adopted in Ref. 35, is used here. It therefore possesses the same difficulty as the Chapman-Enskog expansion: If we proceed to a higher order, the equation corresponding to Eq. (161) becomes a higher-order differential equation for $N$. In contrast to Eq. (127) for small temperature variation, Eq. (161) seemingly 


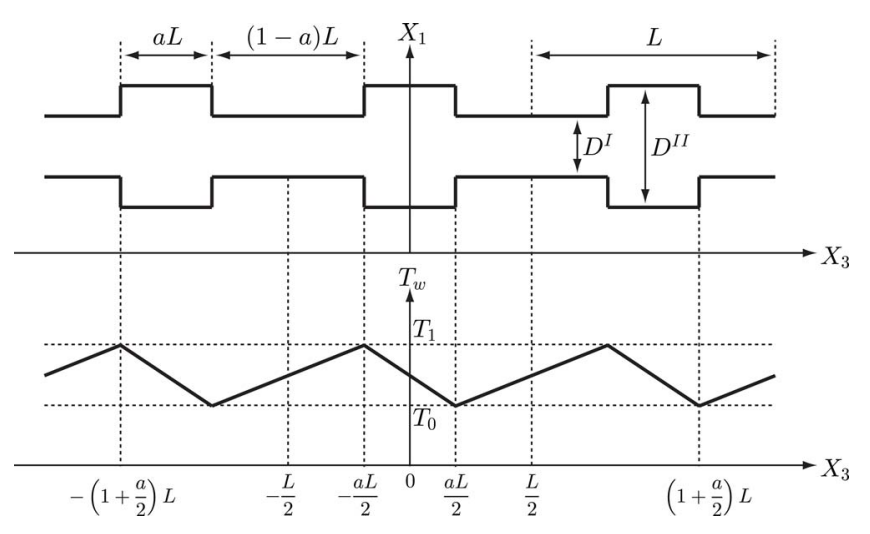

FIG. 3. An example of the Knudsen compressor.

loses the nature of the diffusion equation in the limit $\epsilon \rightarrow 0$. However, the numerical results in Sec. V B show that the gradient $\partial_{y} N$ increases with the decrease of $\epsilon$ (globally or locally depending on the problem), so that the second term of Eq. (161b) remains finite. The comparison with the results based on the nonhomogenized system in Sec. V B shows that Eq. (161) gives excellent results.

\section{NUMERICAL RESULT AND DISCUSSIONS}

In this section, we show some numerical results in the case where the pipe is a two-dimensional channel with an infinite extent in the $X_{2}$ direction, as shown in Fig. 3. To be more specific, the narrow segment is a channel with width $D^{\mathrm{I}}$ and length $(1-a) L$, and the wide segment is a channel with width $D^{\mathrm{II}}$ and length $a L$. The temperature is common to both walls and is a sawtooth distribution as shown in Fig. 3. More precisely,

$T_{w}=\left\{\begin{array}{ll}T_{0}, & \text { at } X_{3}=(a / 2+n) L, \\ T_{1}, & \text { at } X_{3}=(1-a / 2+n) L,\end{array},(n=0, \pm 1, \pm 2, \ldots)\right.$,

and $T_{w}\left(X_{3}\right)$ is a piecewise linear function of $X_{3}$ joining $T_{0}$ and $T_{1}$. We consider the part $X_{3} \in[a L / 2,(a / 2+m) L](m$ $=1,2, \ldots$ ) in the following two situations (Fig. 4):

(A) The channel is closed at $X_{3}=(a / 2+m) L$, and the pressure at the open end $X_{3}=a L / 2$ is kept at $p_{0}$.

(B) Both ends are open, and the pressure at $X_{3}=a L / 2$ and that at $X_{3}=(a / 2+m) L$ are kept at $p_{0}$ and $p_{1}$, respectively.

(A)

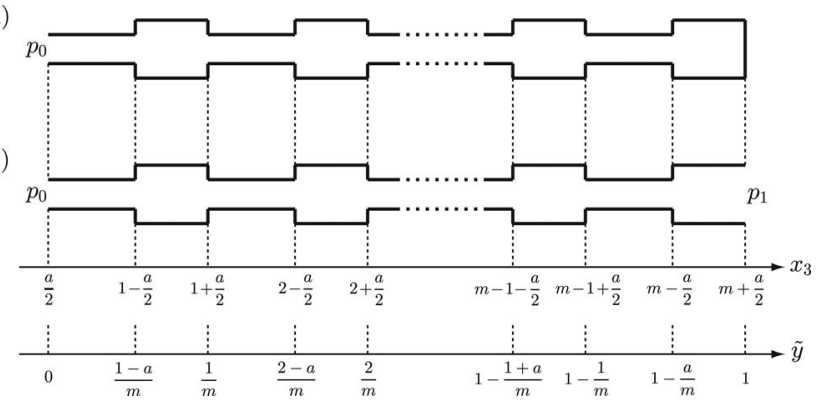

FIG. 4. Knudsen compressor in two different situations: Case (A) and case (B).

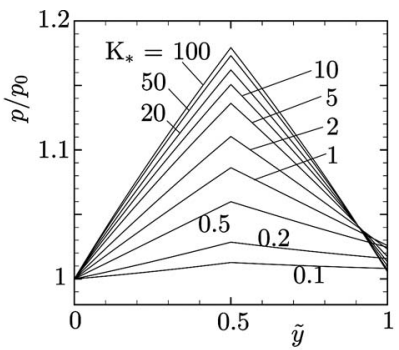

(a)

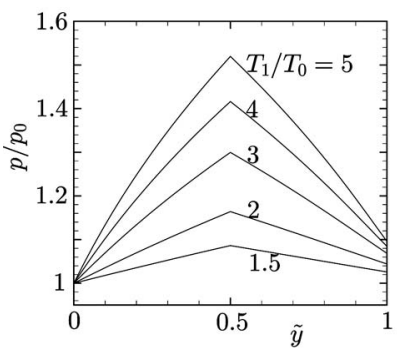

(c)

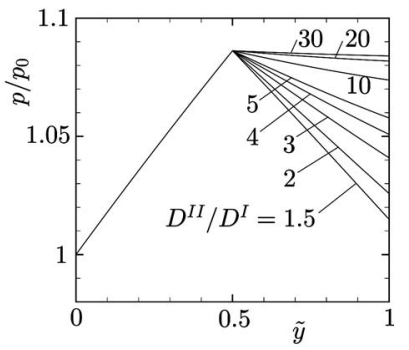

(b)

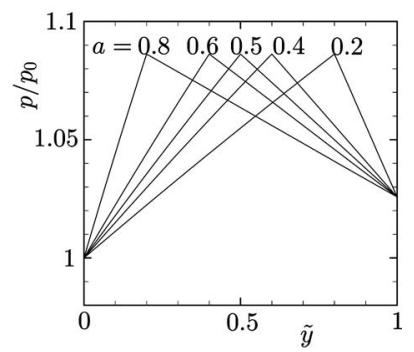

(d)
FIG. 5. $p / p_{0}$ vs $\tilde{y}$ in case (A) $(m=1)$. (a) $D^{\mathrm{II}} / D^{\mathrm{I}}=2, a=0.5, T_{1} / T_{0}=1.5$, and $K_{*}=0.1,0.2,0.5,1,2,5,10,20,50$, and 100. (b) $a=0.5, T_{1} / T_{0}=1.5, K_{*}$ $=1$, and $D^{\mathrm{II}} / D^{\mathrm{I}}=1.5,2,3,4,5,10,20$, and 30 . (c) $D^{\mathrm{II}} / D^{\mathrm{I}}=2, a=0.5, K_{*}$ $=1$, and $T_{1} / T_{0}=1.5,2,3,4$, and 5 . (d) $D^{\mathrm{II}} / D^{\mathrm{I}}=2, K_{*}=1, T_{1} / T_{0}=1.5$, and $a=0.2,0.4,0.5,0.6$, and 0.8 .

We take $p_{0}, T_{0}$, and $D^{\mathrm{I}}$ as the reference pressure $p_{*}$, the reference temperature $T_{*}$, and the reference length $D$, respectively. Therefore, $\hat{p}=1$ at $x_{3}=a / 2$, and $\hat{p}=p_{1} / p_{0}$ at $x_{3}=a / 2$ $+m\left[\right.$ case (B)]; $\hat{T}_{w}\left(x_{3}\right)=1$ at $x_{3}=a / 2+n$, and $\hat{T}_{w}\left(x_{3}\right)=T_{1} / T_{0}$ at $x_{3}=1-a / 2+n$.

We employ the BGK model ${ }^{36,37}$ in place of the Boltzmann equation, Eq. (1) [or Eq. (14)], and assume the diffuse reflection, Eq. (3) with Eq. (9) [or Eq. (18) with Eq. (21)]. In this case, the results in Secs. II-IV undergo slight and inessential modifications, which are summarized in Appendix B. It should be agreed that the equations of Sec. IV referred to in this section have been modified in accordance with Appendix B. In addition, the (dimensionless) cross section $\hat{S}$ should be interpreted as the cross section of the channel per unit width in the $X_{2}$ direction, so that $\hat{S}^{\mathrm{I}}=D^{\mathrm{I}} / D=1$ and $\hat{S}^{\mathrm{II}}$ $=D^{\mathrm{II}} / D=D^{\mathrm{II}} / D^{\mathrm{I}}$, respectively.

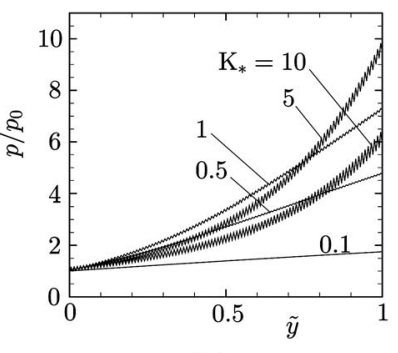

(a)

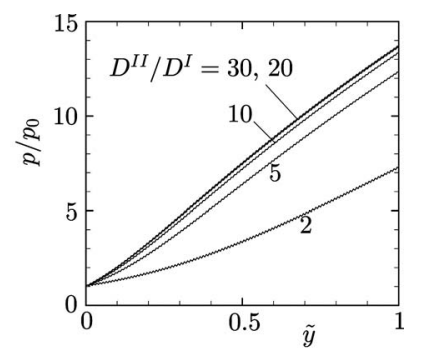

(b)
FIG. 6. $p / p_{0}$ vs $\tilde{y}$ in case (A) $(m=100)$. (a) $D^{\mathrm{II}} / D^{\mathrm{I}}=2, a=0.5, T_{1} / T_{0}=1.5$, and $K_{*}=0.1,0.5,1,5$, and 10 . (b) $a=0.5, T_{1} / T_{0}=1.5, K_{*}=1$, and $D^{\mathrm{II}} / D^{\mathrm{I}}$ $=2,5,10,20$, and 30 . 


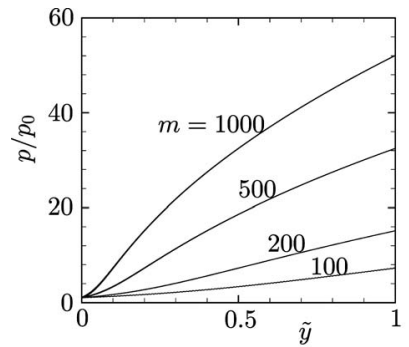

(a)

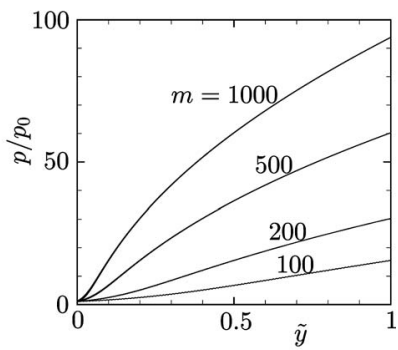

(b)
FIG. 7. $p / p_{0}$ vs $\tilde{y}$ for large $m$ in case (A) $\left(D^{\mathrm{II}} / D^{\mathrm{I}}=2, a=0.5\right.$, and $\left.K_{*}=1\right)$. (a) $T_{1} / T_{0}=1.5$, (b) $T_{1} / T_{0}=2$.

\section{A. Nonhomogenized system}

Let us consider the flow between two infinite plates whose distance is the reference length $D=D^{\mathrm{I}}$ and located at $X_{1}= \pm D / 2= \pm D^{\mathrm{I}} / 2$ or at $x_{1}= \pm 1 / 2$. We suppose that the solution $\phi_{\alpha}^{0}\left(x_{1}, \mathbf{c} ; \tilde{K}\right)$ to Eqs. (B6) and (B7) is known. Then, corresponding to Eqs. (49) and (56), we have

$$
\begin{aligned}
& u_{\alpha}^{0}\left(x_{1} ; \tilde{K}\right)=\int c_{3} \phi_{\alpha}^{0}\left(x_{1}, \mathbf{c} ; \tilde{K}\right) d \mathbf{c}, \\
& M_{\alpha}^{0}(\tilde{K})=\int_{-1 / 2}^{1 / 2} u_{\alpha}^{0}\left(x_{1} ; \tilde{K}\right) d x_{1} .
\end{aligned}
$$

The $M_{\alpha}^{0}$ here and $M_{\alpha}$ appearing in Eq. (168) below are dimensionless mass-flow rate per unit width in the $X_{2}$ direction. The database for $M_{\alpha}^{0}(\tilde{K})$, the original version of which was built by Sone and Itakura, ${ }^{38}$ has been constructed by Sone, Itakura, and Handa. From this, one can obtain an accurate value of $M_{\alpha}^{0}$ instantaneously for an arbitrary Knudsen number $\widetilde{K}$. The database is available from the present authors (the software package can be downloaded from the web-

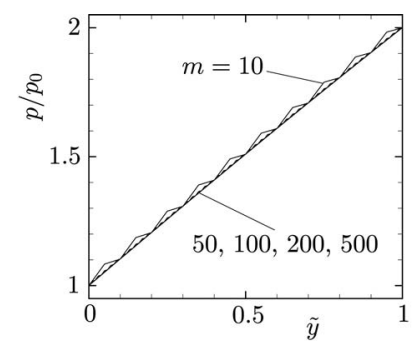

(a)

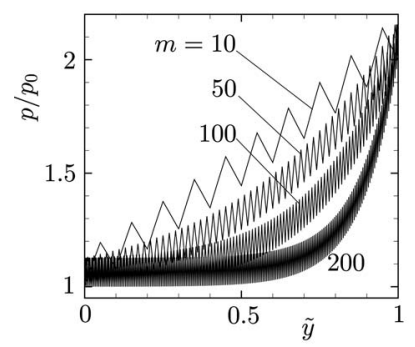

(c)

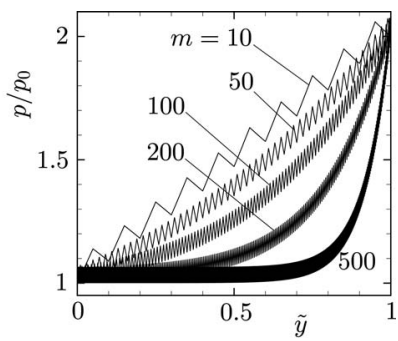

(b)

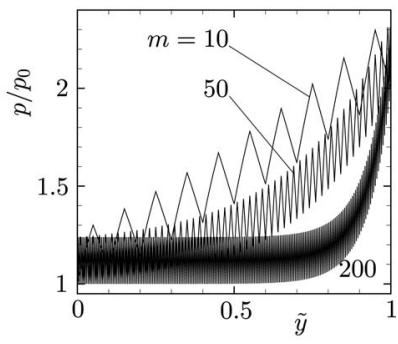

(d)
FIG. 8. $p / p_{0}$ vs $\tilde{y}$ for large $m$ in case (B) $\left(D^{\mathrm{II}} / D^{\mathrm{I}}=2, a=0.5, p_{1} / p_{0}=2\right.$, and $K_{*}=1$ ). (a) $T_{1} / T_{0}=1$, (b) $T_{1} / T_{0}=1.5$, (c) $T_{1} / T_{0}=2$, and (d) $T_{1} / T_{0}=3$.
TABLE I. Dimensionless mass-flow rate $M$ corresponding to Fig. 8 .

\begin{tabular}{rrrrr}
\hline \hline & \multicolumn{4}{c}{$M$} \\
\cline { 2 - 5 }$m$ & $T_{1} / T_{0}=1$ & \multicolumn{1}{c}{$T_{1} / T_{0}=1.5$} & $T_{1} / T_{0}=2$ & \multicolumn{1}{c}{$T_{1} / T_{0}=3$} \\
\hline 10 & $-1.2861 \times 10^{-1}$ & $-7.1009 \times 10^{-2}$ & $-3.7760 \times 10^{-2}$ & $-1.2691 \times 10^{-2}$ \\
20 & $-6.4279 \times 10^{-2}$ & $-1.4913 \times 10^{-2}$ & $1.2897 \times 10^{-2}$ & $4.2663 \times 10^{-2}$ \\
50 & $-2.5705 \times 10^{-2}$ & $-1.7430 \times 10^{-2}$ & $3.9885 \times 10^{-2}$ & $6.2744 \times 10^{-2}$ \\
100 & $-1.2851 \times 10^{-2}$ & $2.6280 \times 10^{-2}$ & $4.5076 \times 10^{-2}$ & $6.4907 \times 10^{-2}$ \\
200 & $-6.4254 \times 10^{-3}$ & $2.8558 \times 10^{-2}$ & $4.5632 \times 10^{-2}$ & $6.4979 \times 10^{-2}$ \\
500 & $-2.5701 \times 10^{-3}$ & $2.8725 \times 10^{-2}$ & $\cdots$ & $\cdots$ \\
\hline \hline
\end{tabular}

page http://www.users.kudpc.kyoto-u.ac.jp/ a51424/Sone /database-e.html). The $M_{\alpha}(\widetilde{K})$ in the narrow and wide segments are expressed in terms of $M_{\alpha}^{0}$ as follows:

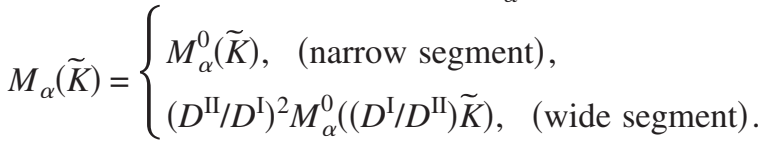

(168)

Then, $Q_{\alpha}(\widetilde{K})$ is defined as in Eqs. (97a) and (97b), that is,

$$
\begin{aligned}
& Q_{P}(\tilde{K})=(1 / \hat{S}) M_{P}(\tilde{K}), \\
& Q_{T}(\widetilde{K})=(1 / \hat{S})\left[M_{P}(\widetilde{K})+M_{T}(\widetilde{K})\right] .
\end{aligned}
$$

With this preparation, we first apply the nonhomogenized system, Eqs. (94)-(96) with the modification $Q_{\alpha}$ $\rightarrow Q_{\alpha}\left(\hat{T}_{w}^{1 / 2} \hat{S} K_{*} / \Pi\right)(\alpha=P, T)$ (see Appendix B), to the present problem. In the present paper, we restrict ourselves to the steady state, so that the system with $\partial_{\hat{t}} \Pi=0$ is solved numerically under the following boundary condition (see Fig. 4):

$\Pi\left(x_{3}=a / 2\right)=1, \quad M\left(x_{3}=a / 2+m\right)=0, \quad$ in case $(\mathrm{A})$,

$\Pi\left(x_{3}=a / 2\right)=1$,

$\Pi\left(x_{3}=a / 2+m\right)=\left(D^{\mathrm{I}} / D^{\mathrm{I}}\right)\left(p_{1} / p_{0}\right), \quad$ in case (B).

The problem is then characterized by the following parameters: $a, D^{\mathrm{II}} / D^{\mathrm{I}}, T_{1} / T_{0}, p_{1} / p_{0}$ [case (B)], $K_{*}$, and $m$. In the following, we use the axial coordinate $\tilde{y}$, normalized by the length of the entire channel, i.e.,

$$
\tilde{y}=\left(x_{3}-a / 2\right) / m,
$$

so that the left end of the channel is located at $\tilde{y}=0$ and its right end at $\tilde{y}=1$.

Some numerical results for the steady pressure distribution $p / p_{0}(=\hat{p})$ along the channel in case (A) are shown in Figs. 5-7. Figure 5 shows $p / p_{0}$ versus $\tilde{y}$ when $m=1$ : Fig. 5(a) shows the effect of $K_{*}$, Fig. 5(b) that of $D^{\mathrm{II}} / D^{\mathrm{I}}$, Fig. 5(c) that of $T_{1} / T_{0}$, and Fig. 5(d) that of $a$. Although only the case of $m=1$ is considered in Fig. 5, the basic features of the effect of the parameters on the pressure rise at the closed end can be observed. That is, the highest pressure rise is attained around $K_{*}=1$; the larger $D^{\mathrm{II}} / D^{\mathrm{I}}$ (or $T_{1} / T_{0}$ ) is, the higher the pressure rise becomes; the pressure rise is almost independent of $a$. Figure 6 contains further investigation of the effect 


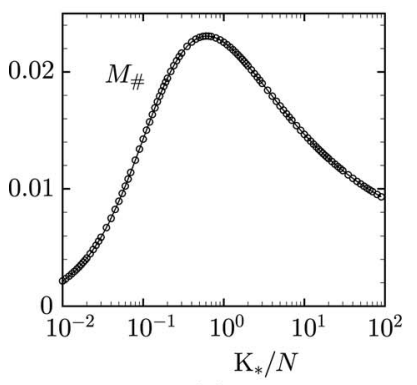

(a)

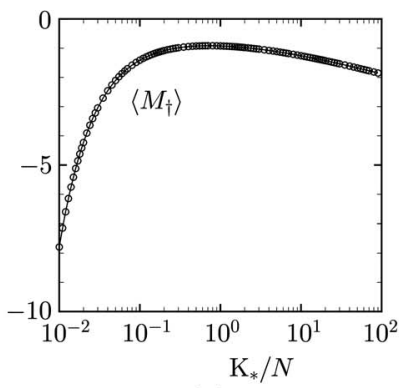

(b)

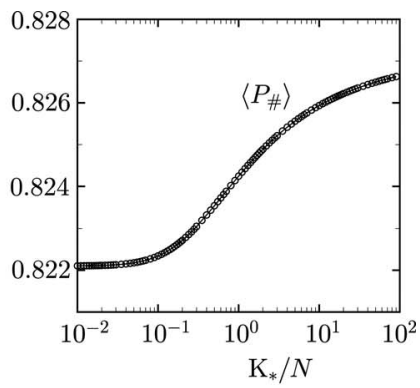

(c)
FIG. 9. $M_{\#},\left\langle M_{\dagger}\right\rangle$, and $\left\langle P_{\#}\right\rangle$ vs $K_{*} / N$ for $T_{1} / T_{0}=1.5\left(D^{\mathrm{II}} / D^{\mathrm{I}}=2, a=0.5\right.$, and $\left.K_{*}=1\right)$. (a) $M_{\#}$, (b) $\left\langle M_{\dot{\dagger}}\right\rangle$, and (c) $\left\langle P_{\#}\right\rangle$. of $K_{*}$ and $D^{\mathrm{II}} / D^{\mathrm{I}}$ on the pressure distribution when 100 units are used $(m=100)$. That is, $p / p_{0}$ versus $\tilde{y}$ is shown for various $K_{*}$ in the case of $D^{\mathrm{II}} / D^{\mathrm{I}}=2, a=0.5$, and $T_{1} / T_{0}=1.5$ in Fig. 6(a), and for various $D^{\mathrm{II}} / D^{\mathrm{I}}$ in the case of $a=0.5$, $T_{1} / T_{0}=1.5$, and $K_{*}=1$ in Fig. 6(b). Among the $K_{*}$ contained in Fig. 6(a), the highest pressure rise at the closed end $(\tilde{y}$ $=1$ ) is attained when $K_{*}=1$. On the other hand, Fig. 6(b) shows that the pressure distribution tends to approach a limiting distribution as $D^{\mathrm{II}} / D^{\mathrm{I}}$ becomes large. The pressure distribution for large numbers of the unit $(m=100,200,500$, and 1000) is shown in Fig. 7, where $D^{\mathrm{II}} / D^{\mathrm{I}}=2, a=0.5, K_{*}$ $=1$, and $T_{1} / T_{0}=1.5$ [Fig. 7(a) $]$ and 2 [Fig. 7(b)]. In the case of $m=1000$, the pressure at the closed end becomes more than 90 times the entrance pressure when $T_{1} / T_{0}=2$.

Figure 8 shows the steady pressure distribution $p / p_{0}$ $(=\hat{p})$ along the channel in case (B) for various $m$ and $T_{1} / T_{0}$ in the case of $a=0.5, D^{\mathrm{II}} / D^{\mathrm{I}}=2, p_{1} / p_{0}=2$, and $K_{*}=1$ : Fig. 8(a) for $T_{1} / T_{0}=1$, Fig. 8(b) for $T_{1} / T_{0}=1.5$, Fig. 8(c) for $T_{1} / T_{0}=2$, and Fig. $8(\mathrm{~d})$ for $T_{1} / T_{0}=3$. The corresponding dimensionless mass-flow rate $M$ [cf. Eqs. (57) and (58)], which is independent of $x_{3}$ (or $\tilde{y}$ ), is shown in Table I. When the temperature of the channel wall is uniform [Fig. 8(a)], a leftward flow is caused by the pressure difference. When the temperature field, which has an effect to drive a rightward flow, is imposed [Figs. 8(b)-8(d)], it reduces the pressuredriven leftward flow, and as the number of the unit $m$ increases, it overcomes the leftward flow and gives rise to a rightward flow. The resulting rightward flow rate is larger for larger temperature ratio $T_{1} / T_{0}$. As $m \rightarrow \infty$, the mass flow rate tends to approach a limiting value for each $T_{1} / T_{0}(M \rightarrow 0$ for $\left.T_{1} / T_{0}=1\right)$. On the other hand, for large $m$, the global pressure rise takes place only in a narrow layer (with respect to $\tilde{y})$ adjacent to the high-pressure end, and the layer becomes thinner as $m$ becomes large.

\section{B. Homogenized system}

Next, we try to apply the homogenized system developed in Sec. IV C to the present problem. The solution procedure is summarized in Sec. IV C 3. The $M_{\#}\left(K_{*} / N\right)$ and $\left\langle M_{\dagger}\right\rangle$, which are necessary for solving Eq. (161), are shown in Figs. 9 and 10, as the functions of $K_{*} / N$, for $D^{\mathrm{II}} / D^{\mathrm{I}}=2$, $a=0.5$, and $K_{*}=1$; Fig. 9 is for $T_{1} / T_{0}=1.5$, and Fig. 10 for $T_{1} / T_{0}=2$. In the figures, we also show $\left\langle P_{\#}\right\rangle$, which is necessary for computing the average pressure $\left\langle\hat{p}_{(0)}\right\rangle$ [cf. Eqs. (163) and (164)].

As described below Eq. (165), the left end of the channel is located at $X_{3}=a L / 2\left(x_{3}=a / 2\right)$ and the right end at $X_{3}$ $=(a / 2+m) L\left(x_{3}=a / 2+m\right)$. Therefore, the total length $L_{g}$ is $L_{g}=m L$, the small parameter $\epsilon$ is $\epsilon=L / L_{g}=1 / m$, and the left and right ends are located at $y=(a / 2) \epsilon$ and $y=(a / 2) \epsilon+1$, respectively [i.e., the range of $y$ is shifted from $-1 / 2 \leq y$ $\leq 1 / 2$ (Sec. IV) to $(a / 2) \epsilon \leq y \leq(a / 2) \epsilon+1]$. If we use $\tilde{y}$ defined by Eq. (171), i.e., $\tilde{y}=y-(a / 2) \epsilon$, then the left and right ends are at $\tilde{y}=0$ and $\tilde{y}=1$. We solve Eq. (161) for steady states $\left(\partial_{\tau} N=0\right)$ under the following boundary condition:

$$
\begin{aligned}
& N(\tilde{y}=0)=N_{0}, \quad \mathcal{M}(\tilde{y}=1)=0, \quad \text { in case }(\mathrm{A}), \\
& N(\tilde{y}=0)=N_{0}, \quad N(\tilde{y}=1)=N_{1}, \quad \text { in case }(\mathrm{B}),
\end{aligned}
$$

where $N_{0}$ and $N_{1}$ are given constants. In our analysis, for convenience in comparing the result with that for the nonhomogenized system, $N_{0}$ and $N_{1}$ are chosen in such a way that the conditions at $\tilde{y}=0$ and $\tilde{y}=1[$ case $(\mathrm{B})]$ are consistent with the corresponding conditions, $\Pi\left(x_{3}=a / 2\right)=1$ and $\Pi\left(x_{3}\right.$ $=a / 2+m)=\left(D^{\mathrm{II}} / D^{\mathrm{I}}\right)\left(p_{1} / p_{0}\right)$, in the nonhomogenized system. For this purpose, we identify $N(y)=\left\langle\Pi_{(0)}\right\rangle$ with the average of $\Pi$ over a period $\left[x_{3}-1 / 2, x_{3}+1 / 2\right]$ around $x_{3}=y / \epsilon$, where $\Pi$ is the corresponding solution of the nonhomogenized sys-

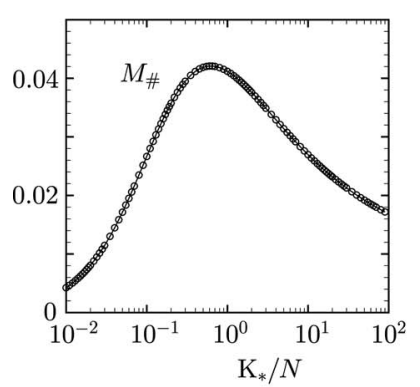

(a)

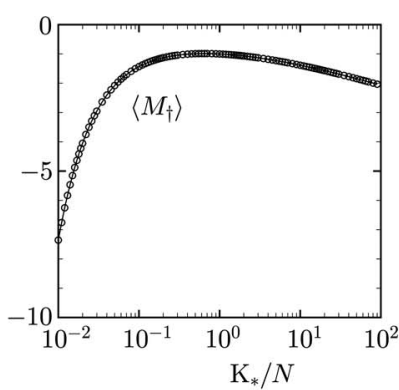

(b)

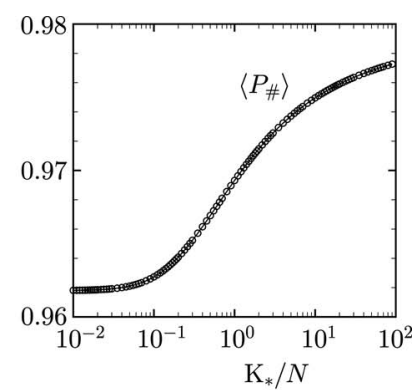

(c)
FIG. 10. $M_{\#},\left\langle M_{\dagger}\right\rangle$, and $\left\langle P_{\#}\right\rangle$ vs $K_{*} / N$ for $T_{1} / T_{0}=2\left(D^{\mathrm{I}} / D^{\mathrm{I}}=2, a=0.5\right.$, and $K_{*}=1$ ). (a) $M_{\#}$, (b) $\left\langle M_{\dagger}\right\rangle$, and (c) $\left\langle P_{\#}\right\rangle$. 


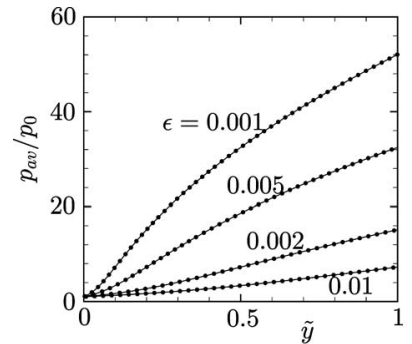

(a)

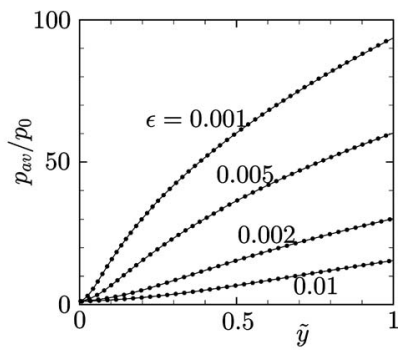

(b)
FIG. 11. $p_{\text {av }} / p_{0}$ vs $\tilde{y}$ for the homogenized system in case (A) $\left(D^{\mathrm{II}} / D^{\mathrm{I}}=2\right.$, $a=0.5$, and $K_{*}=1$ ). (a) $T_{1} / T_{0}=1.5$. (b) $T_{1} / T_{0}=2$. Here, the solid line indicates the result by the homogenized system, and the dots $(\bullet)$ indicate the nonhomogenized system. Note that $\epsilon=1 / \mathrm{m}$.

tem. That is, if we regard $N$ as a function of $\tilde{y}$,

$$
N(\tilde{y})=\left\langle\Pi_{(0)}\right\rangle \simeq \int_{x_{3}-1 / 2}^{x_{3}+1 / 2} \Pi\left(x_{3}^{\prime}\right) d x_{3}^{\prime}=\int_{\tilde{y} / \epsilon+a / 2-1 / 2}^{\tilde{y} / \epsilon+a / 2+1 / 2} \Pi\left(x_{3}^{\prime}\right) d x_{3}^{\prime} .
$$

Note that this $N(\tilde{y})$ is defined only in the interval $a / 2+1 / 2$ $\leq x_{3} \leq a / 2+1 / \epsilon-1 / 2, \quad$ namely, $(1 / 2) \epsilon \leq \tilde{y} \leq 1-(1 / 2) \epsilon$. Therefore, we obtain $N(\tilde{y}=0)$ and $N(\tilde{y}=1)$ by extrapolation and use them as $N_{0}$ and $N_{1}$, respectively.

Some results based on the homogenized system are shown in Figs. 11 and 12. Figure 11, which corresponds to Fig. 7 for the nonhomogenized system, shows the distribution of the average pressure $p_{\mathrm{av}}$, defined by $p_{\mathrm{av}} / p_{0}=\left\langle\hat{p}_{(0)}\right\rangle$, for case (A) for $D^{\mathrm{II}} / D^{\mathrm{I}}=2, a=0.5, K_{*}=1$, and $T_{1} / T_{0}=1.5$ [Fig. 11(a)] and 2 [Fig. 11(b)]. The solid line indicates $p_{\text {av }} / p_{0}$ obtained from the homogenized system, whereas the dot $(\mathbf{\bullet})$ is the corresponding average pressure obtained from the solution of the nonhomogenized system. In both figures, the

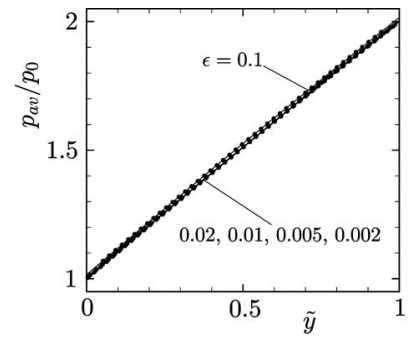

(a)

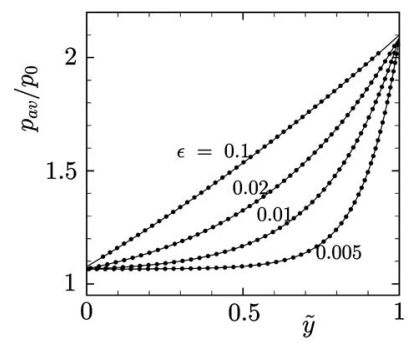

(c)

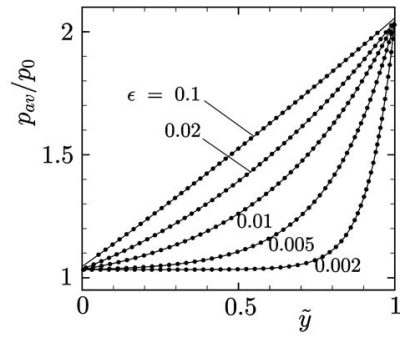

(b)

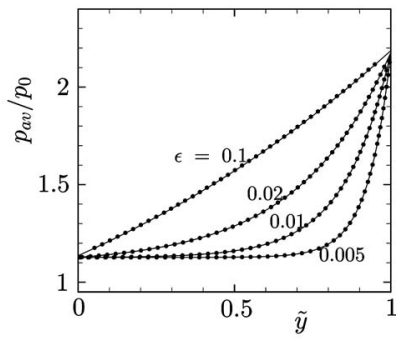

(d)
FIG. 12. $p_{\text {av }} / p_{0}$ vs $\tilde{y}$ for the homogenized system in case (B) $\left(D^{\mathrm{II}} / D^{\mathrm{I}}=2\right.$, $a=0.5, p_{1} / p_{0}=2$, and $K_{*}=1$ ). (a) $T_{1} / T_{0}=1$, (b) $T_{1} / T_{0}=1.5$, (c) $T_{1} / T_{0}=2$, (d) $T_{1} / T_{0}=3$. See the caption of Fig. 11 .
TABLE II. Dimensionless mass-flow rate $\mathcal{M}$ corresponding to Fig. 12 .

\begin{tabular}{lcccc}
\hline \hline \multicolumn{4}{c}{$\mathcal{M}$} \\
\cline { 2 - 5 }$\epsilon$ & $T_{1} / T_{0}=1$ & $T_{1} / T_{0}=1.5$ & $T_{1} / T_{0}=2$ & $T_{1} / T_{0}=3$ \\
\hline 0.1 & $-1.2862 \times 10^{-1}$ & $-7.1056 \times 10^{-2}$ & $-3.7824 \times 10^{-2}$ & $-1.3516 \times 10^{-2}$ \\
0.05 & $-6.4280 \times 10^{-2}$ & $-1.4925 \times 10^{-2}$ & $1.2880 \times 10^{-2}$ & $4.2637 \times 10^{-2}$ \\
0.02 & $-2.5705 \times 10^{-2}$ & $-1.7428 \times 10^{-2}$ & $3.9881 \times 10^{-2}$ & $6.2736 \times 10^{-2}$ \\
0.01 & $-1.2851 \times 10^{-2}$ & $2.6279 \times 10^{-2}$ & $4.5074 \times 10^{-2}$ & $6.4900 \times 10^{-2}$ \\
0.005 & $-6.4254 \times 10^{-3}$ & $2.8558 \times 10^{-2}$ & $4.5630 \times 10^{-2}$ & $6.4973 \times 10^{-2}$ \\
0.002 & $-2.5701 \times 10^{-3}$ & $2.8725 \times 10^{-2}$ & $\ldots$ & $\ldots$ \\
\hline \hline
\end{tabular}

agreement between the results based on the nonhomogenized system and those based on the homogenized system is excellent. Figure 12, which corresponds to Fig. 8 for the nonhomogenized system, shows the distribution of the average pressure $p_{\text {av }}$ for case (B) for $a=0.5, D^{\mathrm{II}} / D^{\mathrm{I}}=2, p_{1} / p_{0}=2, K_{*}$ $=1$, and $T_{1} / T_{0}=1$ [Fig. 12(a)], 1.5 [Fig. 12(b)], 2 [Fig. 12(c)], and 3 [Fig. 12(d)]. Again, the solid line indicates the results obtained from the homogenized system, and the dots (-) are those from the nonhomogenized system. Their agreement is also very good in this figure. Note that $p_{\text {av }} / p_{0}$ is larger than 1 at the left end and larger than 2 at the right end because the bottom value of the sawtooth distribution of $p / p_{0}$ (see Fig. 8) is chosen to be 1 at the left end and to be 2 at the right end. The dimensionless mass-flow rate $\mathcal{M}$, which corresponds to $M$ in the nonhomogenized system [Eqs. (94)-(97)], is shown in Table II for the cases contained in Fig. 12. Thus, Table II corresponds to Table I, and their agreement is excellent.

\section{CONCLUDING REMARKS}

In the present study, we have derived a diffusion model of the Knudsen compressor on the basis of kinetic theory of gases, i.e., the Boltzmann equation and its kinetic boundary condition. The analysis is a formal extension of the previous work, ${ }^{35}$ where a special BGK-type collision model is employed for the purpose of deriving the model with an analytical expression.

As the first step, we investigated rarefied gas flows through a long pipe with a temperature distribution varying slowly in the axial direction and derived a diffusion model that determines the pressure or density distribution along the pipe as well as the mass-flow rate (Sec. II). This model needs information about the mass-flow rate for the (generalized) Poiseuille flow and that for the (generalized) thermal transpiration for the entire range of the Knudsen number, which are available in the literature for some specific models for molecular collisions in the gas and for gas-surface interaction. As the second step, we considered the case where two long pipes with different cross sections are connected at a junction (Sec. III). We applied the diffusion model derived above to each pipe and derived the connection condition at the junction, which consists essentially of the continuity of the pressure and that of the mass flux there. The diffusion model with the connection condition may have potential applicability for gas flows in microchannels. 
Then, we applied the model to a typical configuration of the Knudsen pump, i.e., a long pipe with periodic structure, repetition of a narrow and a wide segment, with a periodic temperature distribution (Sec. IV). Under the assumption that the diffusion model and the connection condition are applicable to each segment, we have derived diffusion models for the overall Knudsen pump with the help of the homogenization technique. Finally, we have shown some numerical examples of the models, using the mass-flow rates of the Poiseuille flow and the thermal transpiration based on the BGK model. The model provides a convenient tool for estimating the performance of the Knudsen pump of various different configurations.

\section{ACKNOWLEDGMENTS}

This work was supported by a JSPS-CNRS joint research program, a PICS project of CNRS, and Grants-in-Aid for Scientific Research from JSPS (No. 17360041) and from MEXT (No. 1765033). It is also supported by the Center of Excellence for Research and Education on Complex Functional Mechanical Systems.

\section{APPENDIX A: UNIQUENESS OF $\hat{\boldsymbol{f}}_{(0)}$}

In this Appendix, we show that the Maxwellian (30) is the unique solution to Eqs. (27) and (28) within the arbitrariness of $\sigma_{(0)}$. If we multiply Eq. (27) by $1+\ln \hat{f}_{(0)}$ and integrate over the whole space of $\boldsymbol{\zeta}$, we have

$$
\nabla_{\underline{x}} \cdot \underline{H}=G \leq 0,
$$

where

$$
\begin{aligned}
\underline{\boldsymbol{H}} & =\int \underline{\boldsymbol{\zeta}} \hat{f}_{(0)} \ln \hat{f}_{(0)} d \boldsymbol{\zeta}, \\
G & =\frac{2}{\sqrt{\pi}} \frac{1}{K_{*}} \int\left(\ln \hat{f}_{(0)}\right) \hat{J}\left(\hat{f}_{(0)}, \hat{f}_{(0)}\right) d \zeta .
\end{aligned}
$$

Here, $G \leq 0$ is the fundamental property of the collision term, and the equality holds if and only if $\hat{f}_{(0)}$ is a local Maxwellian distribution. ${ }^{1-5}$ Integration of Eq. (A1) with respect to $\underline{\mathbf{x}}$ over the cross section $\mathrm{S}$ of the pipe leads to

$$
-\int_{\partial \mathrm{S}} \underline{\boldsymbol{H}} \cdot \mathbf{n} d \gamma=\int_{\mathrm{S}} G d \underline{\mathbf{x}} \leq 0,
$$

where $d \gamma$ is the line element along the pipe wall in the $\left(x_{1}, x_{2}\right)$ plane, and the Gauss theorem has been used. On the other hand, for the boundary condition (28), the following inequality holds:

$$
\underline{\boldsymbol{H}} \cdot \mathbf{n} \leq-\frac{1}{\hat{T}_{w}} \int \mathbf{n} \cdot \underline{\boldsymbol{\zeta}}\left(|\zeta|^{2}-\left|\hat{\mathbf{v}}_{(0)}\right|^{2}\right) \hat{f}_{(0)} d \zeta,
$$

where the equality sign holds if and only if $\hat{f}_{(0)}$ is the Maxwellian satisfying both the Boltzmann equation (27) and the boundary condition (28). Equation (A4) is obtained just by adjusting the estimate obtained by Darrozes and Guiraud ${ }^{65}$ (see also Refs. 2, 4, and 5) to the present problem. Let us multiply Eq. (27) by 1 and $|\zeta|^{2}$ and integrate over the whole space of $\zeta$. Then we have

$$
\begin{aligned}
& \nabla_{\underline{x}} \cdot \int \underline{\zeta} \hat{f}_{(0)} d \zeta=0, \\
& \nabla_{\underline{x}} \cdot \int \underline{\zeta}|\zeta|^{2} \hat{f}_{(0)} d \zeta=0,
\end{aligned}
$$

because of the property of the collision term. Integrating Eq. (A5) with respect to $\underline{\mathbf{x}}$ over the cross section $\mathrm{S}$ of the pipe and using the Gauss theorem, we obtain

$$
\begin{aligned}
& \int_{\partial \mathrm{S}}\left(\int \mathbf{n} \cdot \underline{\zeta} \hat{f}_{(0)} d \zeta\right) d \gamma=0, \\
& \int_{\partial \mathrm{S}}\left(\int \mathbf{n} \cdot \underline{\zeta}|\zeta|^{2} \hat{f}_{(0)} d \zeta\right) d \gamma=0 .
\end{aligned}
$$

Therefore, integration of Eq. (A4) over the cross section gives

$$
\int_{\partial \mathrm{S}} \underline{\boldsymbol{H}} \cdot \mathbf{n} d \gamma \leq 0 .
$$

From Eqs. (A3) and (A7), it follows that

$$
\int_{\partial \mathrm{S}} \underline{\boldsymbol{H}} \cdot \mathbf{n} d \gamma=0
$$

That is, the equality sign holds in Eq. (A3). This means that $\hat{f}_{(0)}$ is a local Maxwellian. Let us put

$$
\hat{f}_{(0)}=\frac{\hat{\rho}_{a}}{\left(\pi \hat{T}_{a}\right)^{3 / 2}} \exp \left(-\frac{\left|\zeta-\hat{\mathbf{v}}_{a}\right|^{2}}{\hat{T}_{a}}\right),
$$

where $\hat{\rho}_{a}, \hat{\mathbf{v}}_{a}$, and $\hat{T}_{a}$ are functions of $\hat{t}$ and $\mathbf{x}$. If we insert Eq. (A9) into Eq. (27), we find that $\hat{\rho}_{a}, \hat{\mathbf{v}}_{a}$, and $\hat{T}_{a}$ are of the following form:

$$
\begin{aligned}
& \hat{\rho}_{a}=a\left(\hat{t}, x_{3}\right) \exp \left(\left|\hat{\mathbf{v}}_{a}\right|^{2} / \hat{T}_{a}\right), \\
& \hat{\mathbf{v}}_{a}=b\left(\hat{t}, x_{3}\right) \mathbf{e}_{3} \times \mathbf{x}+\mathbf{c}\left(\hat{t}, x_{3}\right), \\
& \hat{T}_{a}=d\left(\hat{t}, x_{3}\right),
\end{aligned}
$$

where $a, b, \mathbf{c}$, and $d$ are arbitrary functions of $\hat{t}$ and $x_{3}$, the $\mathbf{e}_{3}$ is the unit vector in the $x_{3}$ direction, and $\mathbf{X}$ indicates the vector product. This solution corresponds to the superposition of a solid-body rotation and a uniform flow. ${ }^{1,5,66}$ From the assumption stated after Eq. (8), the Maxwellian of the form of Eq. (30) is the only Maxwellian [within the arbitrariness of $\left.\sigma_{(0)}\right]$ that satisfies the boundary condition (28). Therefore, Eq. (A9) with Eqs. (A10a)-(A10c) should coincide with Eq. (30) on the pipe wall. It is possible only when $b=\mathbf{c}=0$ and $d=\hat{T}_{w}\left(x_{3}\right)$. This means that Eq. (30) is the unique solution [within the arbitrariness of $\sigma_{(0)}$ ] to Eqs. (27) and (28).

A similar uniqueness proof for the case of the diffusereflection boundary condition is found in Ref. 67, where a 
gas flow in reactive porous media is investigated. The present proof is its extension to a general boundary condition. It should also be mentioned that a similar method has been used to show the uniqueness of a Maxwellian solution in a half space for a general boundary condition. ${ }^{5,68}$

\section{APPENDIX B: CASE OF THE BGK MODEL}

We first go back to the analysis in Sec. II C with the BGK model ${ }^{36,37}$ and the diffuse reflection on the pipe wall. For the BGK model, the $J(f, f)$ in Eq. (1) is replaced by

$$
\begin{aligned}
& J(f, f)=J_{\mathrm{BGK}}(f)=A_{c} \rho\left(f_{e}-f\right), \\
& f_{e}=\frac{\rho}{(2 \pi R T)^{3 / 2}} \exp \left(-\frac{|\boldsymbol{\xi}-\mathbf{v}|^{2}}{2 R T}\right),
\end{aligned}
$$

where $\rho, \mathbf{v}$, and $T$ are given by Eq. (11), and $A_{c}$ is a constant ( $A_{c} \rho$ is the collision frequency of the molecules). For this equation, the mean free path $l_{*}$ is given by

$$
l_{*}=\frac{2}{\sqrt{\pi}} \frac{\left(2 R T_{*}\right)^{1 / 2}}{A_{c} \rho_{*}} .
$$

In this case, $\hat{L}_{\hat{T}_{w}}(\phi)$ in Eq. (38) becomes

$$
\begin{aligned}
& \hat{L}_{\hat{T}_{w}}(\phi)=\hat{T}_{w}^{-1 / 2}[\Psi(\phi)-\phi], \\
& \Psi(\phi)=\omega(\phi)+2 \mathbf{u}(\phi) \cdot \mathbf{c}+\theta(\phi)\left(|\mathbf{c}|^{2}-3 / 2\right), \\
& \omega(\phi)=\int \phi E(\mathbf{c}) d \mathbf{c}, \\
& \mathbf{u}(\phi)=\int \mathbf{c} \phi E(\mathbf{c}) d \mathbf{c}, \\
& \theta(\phi)=(2 / 3) \int\left(|\mathbf{c}|^{2}-3 / 2\right) \phi E(\mathbf{c}) d \mathbf{c} .
\end{aligned}
$$

Therefore, if we introduce a new local Knudsen number $\widetilde{K}$,

$$
\widetilde{K}=\frac{K_{*}\left[\hat{T}_{w}\left(x_{3}\right)\right]^{1 / 2}}{\hat{\rho}_{(0)}\left(\hat{t}, x_{3}\right)}=\frac{K_{*}\left[\hat{T}_{w}\left(x_{3}\right)\right]^{3 / 2}}{\hat{p}_{(0)}\left(\hat{t}, x_{3}\right)},
$$

we can get rid of $\hat{T}_{w}$ from the equation. As a consequence, $\hat{f}_{(1)}$ in Eq. (46) is obtained in the following form:

$$
\begin{aligned}
\hat{f}_{(1)}= & \hat{f}_{(0)}\left[\phi_{P}\left(\underline{\mathbf{x}}, \frac{\zeta}{\hat{T}_{w}^{1 / 2}} ; \tilde{K}\left(\hat{t}, x_{3}\right)\right) \partial_{x_{3}} \ln \hat{p}_{(0)}\right. \\
& \left.+\phi_{T}\left(\underline{\mathbf{x}}, \frac{\zeta}{\hat{T}_{w}^{1 / 2}} ; \tilde{K}\left(\hat{t}, x_{3}\right)\right) \partial_{x_{3}} \ln \hat{T}_{w}\right] .
\end{aligned}
$$

Here, $\phi_{\alpha}(\underline{\mathbf{x}}, \mathbf{c} ; \tilde{K})(\alpha=P, T)$ is the solution to the equation

$$
\underline{\mathbf{c}} \cdot \nabla_{\underline{x}} \phi_{\alpha}=\frac{2}{\sqrt{\pi}} \frac{1}{\widetilde{K}}\left[\Psi\left(\phi_{\alpha}\right)-\phi_{\alpha}\right]-\mathrm{I}_{\alpha}
$$

where $\mathrm{I}_{\alpha}$ is given by Eq. (45), and the boundary condition

$$
\begin{gathered}
\phi_{\alpha}(\underline{\mathbf{x}}, \mathbf{c} ; \tilde{K})=-2 \sqrt{\pi} \int_{\mathbf{c}_{*} \cdot \mathbf{n}<0} \mathbf{c}_{*} \cdot \mathbf{n} \phi_{\alpha}\left(\underline{\mathbf{x}}, \mathbf{c}_{*} ; \tilde{K}\right) E\left(\mathbf{c}_{*}\right) d \mathbf{c}_{*}, \\
\text { for } \mathbf{c} \cdot \mathbf{n}>0, \underline{\mathbf{x}} \in \partial \mathrm{S},
\end{gathered}
$$

where $k_{\hat{T}_{w}}=-2 \sqrt{\pi} \mathbf{c}_{*} \cdot \mathbf{n}$ for the diffuse reflection has been used [see the sentence following Eq. (40g)]. Note that $\phi_{\alpha}$ is affected by $\hat{T}_{w}$ only through $\widetilde{K}$. Then, corresponding to Eqs. (49) and (56), we obtain $u_{\alpha}(\underline{\mathbf{x}} ; \tilde{K})$ and $M_{\alpha}(\tilde{K} ; \mathrm{S})$. We must use these $M_{P}$ and $M_{T}$, i.e., $M_{P}\left(\hat{T}_{w}^{3 / 2} K_{*} / \hat{p}_{(0)} ; \mathrm{S}\right) \quad$ and $M_{T}\left(\hat{T}_{w}^{3 / 2} K_{*} / \hat{p}_{(0)} ; \mathrm{S}\right)$, in Eq. (57b). Correspondingly, $Q_{P}$ and $Q_{T}$ in Eqs. (97a) and (97b) become $Q_{P}\left(\hat{T}_{w}^{1 / 2} \hat{S} K_{*} / \Pi ; \mathrm{S}\right)$ and $Q_{T}\left(\hat{T}_{w}^{1 / 2} \hat{S} K_{*} / \Pi ; \mathrm{S}\right)$, which should be used throughout Sec. IV. In particular, the following replacement should be made: For $\alpha=P, T$,

$Q_{\alpha \#} \rightarrow Q_{\alpha}\left(\frac{\hat{T}_{w}^{1 / 2} \hat{S}}{\prod_{\#}} \frac{K_{*}}{N} ; \mathrm{S}\right), \quad$ in Eqs. (139) and (156),

$\partial_{2} Q_{\alpha \#} \rightarrow \hat{T}_{w}^{1 / 2} Q_{\alpha}^{\prime}\left(\frac{\hat{T}_{w}^{1 / 2} \hat{S}}{\prod_{\#}} \frac{K_{*}}{N} ; \mathrm{S}\right), \quad$ in Eq. (156),

where ' indicates the derivative with respect to the argument, i.e., $Q_{\alpha}^{\prime}(x ; \mathrm{S})=(d / d x) Q_{\alpha}(x ; \mathrm{S})$.

${ }^{1}$ M. N. Kogan, Rarefied Gas Dynamics (Plenum, New York, 1969).

${ }^{2} \mathrm{C}$. Cercignani, The Boltzmann Equation and its Applications (SpringerVerlag, Berlin, 1988).

${ }^{3}$ C. Cercignani, Rarefied Gas Dynamics: From Basic Concepts to Actual Calculations (Cambridge University Press, Cambridge, 2000).

${ }^{4}$ Y. Sone, Kinetic Theory and Fluid Dynamics (Birkhäuser, Boston, 2002).

${ }^{5}$ Y. Sone, Molecular Gas Dynamics: Theory, Techniques, and Applications (Birkhäuser, Boston, 2006).

${ }^{6}$ G. M. Karniadakis and A. Beskok, Micro Flows: Fundamentals and Simulation (Springer-Verlag, New York, 2002).

${ }^{7}$ G. Karniadakis, A. Beskok, and N. Aluru, Microflows and Nanoflows: Fundamentals and Simulation (Springer Science+Business Media, New York, 2005).

${ }^{8}$ C. Cercignani, Slow Rarefied Flows: Theory and Application to MicroElectro-Mechanical Systems (Birkhäuser, Basel, 2006).

${ }^{9}$ N. G. Hadjiconstantinou, "The limits of Navier-Stokes theory and kinetic extensions for describing small-scale gaseous hydrodynamics," Phys. Fluids 18, 111301 (2006).

${ }^{10}$ E. H. Kennard, Kinetic Theory of Gases (McGraw-Hill, New York, 1938).

${ }^{11}$ Y. Sone, "Thermal creep in rarefied gas," J. Phys. Soc. Jpn. 21, 1836 (1966).

${ }^{12}$ Y. Sone and K. Yamamoto, "Flow of rarefied gas through a circular pipe," Phys. Fluids 11, 1672 (1968).

${ }^{13}$ T. Ohwada, Y. Sone, and K. Aoki, "Numerical analysis of the shear and thermal creep flows of a rarefied gas over a plane wall on the basis of the linearized Boltzmann equation for hard-sphere molecules," Phys. Fluids A 1, 1588 (1989).

${ }^{14}$ T. Ohwada, Y. Sone, and K. Aoki, "Numerical analysis of the Poiseuille and thermal transpiration flows between two parallel plates on the basis of the Boltzmann equation for hard-sphere molecules," Phys. Fluids A 1, 2042 (1989).

${ }^{15}$ Y. Sone, "Flow induced by thermal stress in rarefied gas," Phys. Fluids 15, 1418 (1972)

${ }^{16} \mathrm{Y}$. Sone, "Flows induced by temperature fields in a rarefied gas and their ghost effect on the behavior of a gas in the continuum limit," in Annual Review of Fluid Mechanics (Annual Reviews, Palo Alto, 2000), p. 779.

${ }^{17}$ M. N. Kogan, V. S. Galkin, and O. G. Fridlender, "Stresses produced in gases by temperature and concentration inhomogeneities: New type of free convection," Sov. Phys. Usp. 19, 420 (1976).

${ }^{18} \mathrm{~K}$. Aoki, Y. Sone, and N. Masukawa, "A rarefied gas flow induced by a temperature field," in Rarefied Gas Dynamics, edited by J. Harvey and G. Lord (Oxford University Press, Oxford, 1995), p. 35. 
${ }^{19}$ Y. Sone and M. Yoshimoto, "Demonstration of a rarefied gas flow induced near the edge of a uniformly heated plate," Phys. Fluids 9, 3530 (1997).

${ }^{20} \mathrm{H}$. Sugimoto and Y. Sone, "Vacuum pump without a moving part by thermal edge flow," in Rarefied Gas Dynamics, edited by M. Capitelli (AIP, Melville, 2005), p. 168.

${ }^{21}$ M. Knudsen, "Eine revision der gleichgewichtsbedingung der gase. Thermische molekularströmung," Ann. Phys. 31, 205 (1910).

${ }^{22}$ M. Knudsen, "Thermischer molekulardruck der gase in Röhren," Ann. Phys. 33, 1435 (1910).

${ }^{23}$ G. Pham-Van-Diep, P. Keeley, E. P. Muntz, and D. P. Weaver, "A micromechanical Knudsen compressor," in Rarefied Gas Dynamics, edited by J. Harvey and G. Lord (Oxford University Press, Oxford, 1995), p. 715.

${ }^{24}$ Y. Sone, Y. Waniguchi, and K. Aoki, "One-way flow of a rarefied gas induced in a channel with a periodic temperature distribution," Phys. Fluids 8, 2227 (1996).

${ }^{25}$ S. E. Vargo and E. P. Muntz, "An evaluation of a multiple-stage micromechanical Knudsen compressor and vacuum pump," in Rarefied Gas Dynamics, edited by C. Shen (Peking University Press, Peking, 1997), p. 995.

${ }^{26}$ M. L. Hudson and T. J. Bartel, "DSMC simulation of thermal transpiration and accommodation pump," in Rarefied Gas Dynamics, Vol. 1, edited by R. Brun, R. Campargue, R. Gatignol, J.-C. Lengrand (Cépaduès-Éditions, Toulouse, 1999), p. 719.

${ }^{27}$ Y. Sone and K. Sato, "Demonstration of a one-way flow of a rarefied gas induced through a pipe without average pressure and temperature gradients," Phys. Fluids 12, 1864 (2000).

${ }^{28}$ K. Aoki, Y. Sone, S. Takata, K. Takahashi, and G. A. Bird, "One-way flow of a rarefied gas induced in a circular pipe with a periodic temperature distribution," in Rarefied Gas Dynamics, edited by T. J. Bartel and M. A. Gallis (AIP, Melville, 2001), p. 940.

${ }^{29}$ Y. Sone, T. Fukuda, T. Hokazono, and H. Sugimoto, "Experiment on a one-way flow of a rarefied gas through a straight circular pipe without average temperature and pressure gradients," in Rarefied Gas Dynamics, edited by T. J. Bartel and M. A. Gallis (AIP, Melville, 2001), p. 948.

${ }^{30}$ Y. Sone and H. Sugimoto, "Vacuum pump without a moving part and its performance," in Rarefied Gas Dynamics, edited by E. P. Muntz and A. Ketsdever (AIP, Melville, 2003), p. 1041.

${ }^{31}$ Y. L. Han, M. Young, E. P. Muntz, and G. Shiflett, "Knudsen compressor performance at low pressures," in Rarefied Gas Dynamics, edited by M. Capitelli (AIP, Melville, 2005), p. 162.

${ }^{32}$ M. Young, Y. L. Han, E. P. Muntz, and G. Shiflett, "Characterization and optimization of a radially driven multi-stage Knudsen compressor," in Rarefied Gas Dynamics, edited by M. Capitelli (AIP, Melville, 2005), p. 174.

${ }^{33}$ G. A. Bird, Molecular Gas Dynamics (Oxford University Press, Oxford, 1976).

${ }^{34}$ G. A. Bird, Molecular Gas Dynamics and the Direct Simulation of Gas Flows (Oxford University Press, Oxford, 1994).

${ }^{35} \mathrm{~K}$. Aoki and P. Degond, "Homogenization of a flow in a periodic channel of small section," Multiscale Model. Simul. 1, 304 (2003).

${ }^{36}$ P. L. Bhatnagar, E. P. Gross, and M. Krook, "A model for collision processes in gases. I. Small amplitude processes in charged and neutral onecomponent systems," Phys. Rev. 94, 511 (1954).

${ }^{37} \mathrm{P}$. Welander, "On the temperature jump in a rarefied gas," Ark. Fys. 7, 507 (1954).

${ }^{38}$ Y. Sone and E. Itakura, "Analysis of Poiseuille and thermal transpiration flows for arbitrary Knudsen numbers by a modified Knudsen number expansion and their database," J. Vac. Soc. Jpn. 33, 92 (1990) (in Japanese).

${ }^{39} \mathrm{~S}$. Takata, H. Sugimoto, and S. Kosuge, "Gas separation by means of the Knudsen compressor,” Eur. J. Mech. B/Fluids 26, 155 (2007).

${ }^{40} \mathrm{C}$. Cercignani and A. Daneri, "Flow of a rarefied gas between two parallel plates," J. Appl. Phys. 34, 3509 (1963).

${ }^{41}$ C. Cercignani and F. Sernagiotto, "Cylindrical Poiseuille flow of a rarefied gas," Phys. Fluids 9, 40 (1966).

${ }^{42}$ S. K. Loyalka, T. S. Storvick, and H. S. Park, "Poiseuille flow and thermal creep flow in long, rectangular channels in the molecular and transition flow regimes," J. Vac. Sci. Technol. 13, 1188 (1976).

${ }^{43}$ M. Hasegawa and Y. Sone, "Poiseuille and thermal transpiration flows of a rarefied gas for various pipes," J. Vac. Soc. Jpn. 31, 416 (1988) (in Japanese).

${ }^{44}$ S. K. Loyalka, "Thermal transpiration in a cylindrical tube," Phys. Fluids 12, 2301 (1969).

${ }^{45} \mathrm{H}$. Niimi, "Thermal creep flow of rarefied gas between two parallel plates,” J. Phys. Soc. Jpn. 30, 572 (1971).

${ }^{46}$ F. Sharipov and V. Seleznev, "Data on internal rarefied gas flows," J. Phys. Chem. Ref. Data 27, 657 (1998).

${ }^{47}$ F. Sharipov, "Application of the Cercignani-Lampis scattering kernel to calculations of rarefied gas flows. I. Plane flow between two parallel plates," Eur. J. Mech. B/Fluids 21, 113 (2002).

${ }^{48}$ C. E. Siewert, "The linearized Boltzmann equation: Concise and accurate solutions to basic flow problems," ZAMP 54, 273 (2003).

${ }^{49}$ C.-C. Chen, I.-K. Chen, T.-P. Liu, and Y. Sone, "Thermal transpiration for the linearized Boltzmann equation," Commun. Pure Appl. Math. 60, 147 (2007).

${ }^{50}$ S. Fukui and R. Kaneko, "Analysis of ultra-thin gas film lubrication based on linearized Boltzmann equation including thermal creep flow," J. Tribol. 110, 253 (1988).

${ }^{51}$ F. Sharipov, "Rarefied gas flow through a long tube at any temperature ratio," J. Vac. Sci. Technol. A 14, 2627 (1996).

${ }^{52} \mathrm{C}$. Shen, "Use of the degenerated Reynolds equation in solving the microchannel flow problem," Phys. Fluids 17, 046101 (2005).

${ }^{53}$ C. Cercignani, M. Lampis, and S. Lorenzani, "Flow of a rarefied gas between parallel and almost parallel plates," in Rarefied Gas Dynamics, edited by M. Capitelli (AIP, Melville, 2005), p. 719.

${ }^{54}$ H. Babovsky, "On Knudsen flows within thin tube," J. Stat. Phys. 44, 865 (1986).

${ }^{55} \mathrm{C}$. Bardos, H. Babovsky, and T. Platkowski, "Diffusion approximation for a Knudsen gas in a thin domain with accommodation on the boundary," Asymptotic Anal. 3, 265 (1991).

${ }^{56}$ C. Börgers, C. Greengard, and E. Thomann, "The diffusion limit of free molecular flow in thin plate channels," SIAM J. Appl. Math. 52, 1057 (1992).

${ }^{57}$ F. Golse, "Anomalous diffusion limit for the Knudsen gas," Asymptotic Anal. 17, 1 (1998).

${ }^{58} \mathrm{P}$. Degond, "A model of near-wall conductivity and its application to plasma thrusters," SIAM J. Appl. Math. 58, 1138 (1998).

${ }^{59}$ P. Degond, V. Latocha, L. Guarrigues, and J. P. Boeuf, "Electron transport in stationary plasma thrusters," Transp. Theory Stat. Phys. 27, 203 (1998).

${ }^{60}$ V. Latocha, L. Garrigues, P. Degond, and J. P. Boeuf, "Numerical simulation of electron transport in the channel region of a stationary plasma thruster," Plasma Sources Sci. Technol. 11, 104 (2002).

${ }^{61} \mathrm{P}$. Degond and S. Mancini, "Diffusion driven by collisions with the boundary," Asymptotic Anal. 27, 47 (2001).

${ }^{62}$ P. Degond, V. Latocha, S. Mancini, and A. Mellet, "Diffusion dynamics of an electron gas confined between two plates," Methods Appl. Anal. 9, 127 (2002).

${ }^{63} \mathrm{H}$. Grad, "Principles of the kinetic theory of gases," in Handbuch der Physik, edited by S. Flügge (Springer-Verlag, Berlin, 1958), Band XII, p. 205.

${ }^{64} \mathrm{P}$. Degond, "Macroscopic limits of the Boltzmann equation: A review," in Modeling and Computational Methods for Kinetic Equations, edited by P. Degond, L. Pareschi, and G. Russo (Birkhäuser, Boston, 2004), p. 3.

${ }^{65}$ J. S. Darrozes and J.-P. Guiraud, "Généralisation formelle du théorème H en présence de parois. Applications," C.R. Acad. Sci., Paris A 262, 1368 (1966).

${ }^{66} \mathrm{H}$. Grad, "On the kinetic theory of rarefied gases," Commun. Pure Appl. Math. 2, 331 (1949).

${ }^{67}$ P. Charrier and B. Dubroca, "Asymptotic transport models for heat and mass transfer in reactive porous media," Multiscale Model. Simul. 2, 124 (2003).

${ }^{68} \mathrm{C}$. Bardos, F. Golse, and Y. Sone, "Half-space problems for the Boltzmann equation: A survey," J. Stat. Phys. 124, 275 (2006). 